\title{
Local Quasiequivalence and Adiabatic Vacuum States
}

\author{
Christian Lüders and John E. Roberts \\ Fachbereich Physik, Universität Osnabrück, Postfach 4469, W-4500 Osnabrück, \\ Federal Republic of Germany
}

Received December 20, 1989

\begin{abstract}
The problem of determining the physically relevant states acquires a new dimension in curved spacetime where there is, in general, no natural definition of a vacuum state. It is argued that there is a unique local quasiequivalence class of physically relevant states and it is shown how this class can be specified for the free Klein-Gordon field on a Robertson-Walker spacetime by using the concept of an adiabatic vacuum state. Any two adiabatic vacuum states of order two are locally quasiequivalent.
\end{abstract}

\section{Introduction}

In quantum field theory on Minkowski space the vacuum state is of primary importance. It is a state which is invariant under the group of isometries of Minkowski space time, the Poincare group. Furthermore it is a ground state, i.e. a state of lowest energy in the corresponding vacuum representation. On an arbitrary Lorentzian spacetime there are in general no isometries so that there is no corresponding way of trying to define a "vacuum" state. Much work has been devoted to the problem of developing other criteria leading to a suitable definition of a vacuum state. It seems probable that there is no unique natural state for a quantum field theory on an arbitrary Lorentz spacetime which deserves the epithet "vacuum state" but at best some class of states which might represent local equilibrium states.

In view of this situation, it is worth reexamining the reasons which lead one to ask for such a vacuum state. The original motivation was undoubtedly to be able to define a suitable notion of particle. The notion of particle even in a Minkowski quantum field theory is a complex one with several facets which we do not wish to examine in detail here. For our purposes, it will be enough to adopt what is for practical purposes by far the most important notion of particle, namely that which underlies scattering theory. Roughly speaking, a particle is an excitation of the vacuum which is relatively well localized in space and time and moves on a straight line path. The importance of the concept of particle is that it allows one 
to analyse scattering experiments. This is best expressed as the postulate of asymptotic completeness. Every state of relevance to elementary particle physics admits an asymptotic interpretation for $t \rightarrow+\infty$ in terms of a finite set of particles which are well separated from one another and are moving away from each other on straight line paths. The point we wish to make here is that the concept of particle is an asymptotic one which reflects the tendency of localized concentrations of energy to split up asymptotically into a finite number of localized fragments with a simple asymptotic motion and no interaction in the future. The question of whether such a concept is useful in a curved space time is therefore primarily a question of whether we can expect such a similar behaviour in a curved spacetime. In regions of high curvature one would not expect such behaviour because the presence of the gravitating matter needed to produce this high curvature means that the system still has an appreciable interaction. Thus we can only expect to apply the concepts of scattering theory successfully if the spacetime in question is asymptotically flat. As is well known under these circumstances we have no difficulty in defining an asymptotic out vacuum or the associated asymptotic particle states. Hence the importance of the particle concept in physics in no way suggests that one should even try to define a vacuum state in curved spacetime different from the above asymptotic out or in vacuum.

This does not mean that we should conclude that, since we do not need to specify a vacuum state, all states should be treated as equally relevant to a description of the physical world. It is well known that systems with an infinite number of degrees of freedom possess a myriad of states which have not been classified in any sensible manner and that it would be unwise to regard more than a small fraction of these as being relevant to physics. Indeed our experience with quantum field theory in Minkowski space suggests that as far as measurements in some fixed bounded region $\mathcal{O}$ go the situation is extremely simple. It is enough to know one physical state $\omega$ of our system, construct the corresponding GNSrepresentation $\pi_{\omega}$ on the Hilbert space $\mathscr{H}_{\omega}$ and look at the set of expectation values for observables which can be measured in $\mathcal{O}$ given by density matrices on $\mathscr{H}_{\omega}$. This set of expectation values is independent of the choice of the physical state $\omega$.

It has been suggested by Haag, Narnhofer and Stein [1] that this should be true of a quantum field theory in a curved spacetime too and they have called this the principle of local definiteness ${ }^{1}$ and have used this principle to derive the value of the Hawking temperature. If one accepts this principle then it is an important task to specify the class of physical local states for each bounded open set $\mathcal{O}$, and to do so it is enough to specify a single (global) physical state. Alternatively, we can specify some family of physical states, but we then have the task of verifying that the family is consistent with the principle of local definiteness.

In this paper, we examine the free Klein-Gordon field on Robertson-Walker spacetimes and find that we can indeed specify a family of quasifree Fock states which is consistent with the principle of local definiteness. Our scheme is based on a precise version of the notion of adiabatic vacuum. We work in the algebraic

\footnotetext{
1 There has been an unfortunate tendency in the literature to equate the criteria of [1] with the existence of the massless free field scaling limit used in the derivation of the Hawking temperature. The principle of local definiteness does not merely have implications for the scaling limit but restricts the physical states of the full theory
} 
formulation of quantum field theory [2] and construct following Dimock [3] a net $\mathcal{O} \rightarrow \mathfrak{A}(\mathcal{O})$ of $C^{*}$-algebras defined on a suitable class $\mathscr{K}$ of open sets with compact closure associated with a free Klein-Gordon field on a Lorentzian spacetime. Given any state $\omega$ on the net $\mathfrak{U}$, the GNS-construction gives us a representation $\pi_{\omega}$ of the net on a Hilbert space $\mathscr{H}_{\omega}$. The restriction of this representation to the algebra $\mathfrak{U}(\mathcal{O})$ will be denoted $\pi_{\omega} \uparrow \mathcal{O}$. Two states $\omega$ and $\omega^{\prime}$ are said to be locally quasiequivalent if the representations $\pi_{\omega} \uparrow \mathcal{O}$ and $\pi_{\omega^{\prime}} \uparrow \mathcal{O}$ are quasiequivalent for each $\mathcal{O} \in \mathscr{K}$. Equivalently $\omega$ and $\omega^{\prime}$ are locally quasiequivalent if the density matrices in $\mathscr{H}_{\omega}$ and $\mathscr{H}_{\omega^{\prime}}$ define the same set of states of $\mathfrak{U}(\mathcal{O})$ for each $\mathcal{O} \in \mathscr{K}$. The principle of local definiteness holds if any two physical states of $\mathfrak{U}$ are locally quasiequivalent. Our task is therefore to use geometric data to specify a local quasiequivalence class of states on $\mathfrak{U}$.

To fix our notation we sketch the construction of the net $\mathscr{U}$ for the Klein-Gordon field $\varphi$ in a globally hyperbolic manifold $\mathscr{M}$ with Lorentzian metric $g$. The natural choice of test functions for $\varphi$ is the set of complex-valued odd smooth 4 -forms with compact support which we denote by $\mathscr{D}(\mathscr{M})$. The commutation relations are determined by the Green's function

$$
G(x, y):=-i[\varphi(x), \varphi(y)]
$$

which is obtained as follows [3]: there are unique operators $E^{ \pm}: \mathscr{D}(\mathscr{M}) \rightarrow C^{\infty}(\mathscr{M})$ with the property that

$$
\begin{aligned}
& \left(\square+m^{2}\right) E^{ \pm}=E^{ \pm}\left(\square+m^{2}\right)=\#, \\
& \operatorname{supp} E^{ \pm} f \subset J^{ \pm}(\operatorname{supp} f),
\end{aligned}
$$

where \# denotes the Hodge \#-operation and $J^{ \pm}(\operatorname{supp} f)$ denotes the set of points which can be reached from supp $f$ by a future or past directed causal curve, respectively. Setting $E:=E^{+}-E^{-}$, we have $\left(\square+m^{2}\right) E=E\left(\square+m^{2}\right)=0$ and $\int g \wedge E f=-\int f \wedge E g$ and $G$ is given by

$$
G(f, g)=-\int f \wedge E g, \quad f, g \in \mathscr{D}(\mathscr{M}) .
$$

We equip $\mathscr{D}(\mathscr{M})$ with the antilinear involution $\Gamma$ given by $\Gamma f=\bar{f}$ and an Hermitian form $\gamma$ given by $\gamma(f, g)=i G(\bar{f}, g)$. The triple $(\mathscr{D}(\mathscr{M}), \Gamma, \gamma)$ is a phase space in the sense of [4] and there is an associated self-dual CCR-algebra. This algebra has two drawbacks, its representations are by unbounded operators and the corresponding fields do not necessarily satisfy the Klein-Gordon equation. The second problem is cured by replacing $\mathscr{D}(\mathscr{M})$ by its quotient space $\mathscr{D}(\mathscr{M}) /\left(\square+m^{2}\right) \mathscr{D}(\mathscr{M}):=K$ and the induced phase space $(K, \Gamma, \gamma)$ has the additional merit of being non-degenerate. We denote the element of the self-dual CCR-algebra $\mathfrak{A}(K, \Gamma, \gamma)$ corresponding to $f \in \mathscr{D}(\mathscr{M})$ by $\varphi(f)$. The first problem is cured by passing to the associated Weyl algebra defined over the real linear space $\operatorname{Re} K:=\{k \in K: \Gamma k=k\}$ equipped with the symplectic form induced on $\operatorname{Re} K$ by $-i \gamma$. Since the symplectic form is non-degenerate the Weyl algebra is a simple $C^{*}$-algebra. If $W(f)$ denotes the Weyl operator corresponding to $f \in \mathscr{D}(\mathscr{M})$ then we have the commutation relations

$$
W(f) W(g)=W(f+g) e^{-(1 / 2) \gamma(f, g)} .
$$

We get our net $\mathfrak{A}$ of $C^{*}$-algebras associated with the Klein-Gordon field over $\mathscr{M}$ 
by taking $\mathfrak{A}(\mathcal{O})$ to be the $C^{*}$-subalgebra of our Weyl algebra generated by the $W(f)$ with supp $f \subset \mathcal{O}$.

There is a good reason to be dissatisfied with taking any net $\mathfrak{U}$ of Weyl algebras as the observable net of a quantum field theory since there are representations $\pi$ for which $\lambda \mapsto \pi(W(\lambda f))$ is not a strongly continuous 1-parameter unitary group and it is usual to restrict oneselves to representations, known as regular representations, where this does not happen. However, once one has determined the appropriate local quasiequivalence class of states, one can redefine the net to be the associated net of local von Neumann algebras and $\lambda \mapsto \pi(W(\lambda f))$ will be strongly continuous in any locally normal representation.

In the case of free fields in curved spacetime, the quasifree states form a distinguished and simple class of states so that it is natural to try and specify the local quasiequivalence class of physical states by singling out a special class of quasifree states. This special class will be the adiabatic vacuum states introduced in Sect. 3.

A quasifree state $\omega_{S}$ is specified by its 2-point function which is a scalar product on the test function space $\mathscr{D}(\mathscr{M})$ or, more precisely on $K$,

$$
S(f, g):=\omega_{S}\left(\varphi(f)^{*} \varphi(g)\right) .
$$

In terms of the Weyl operators we have

$$
\omega_{S}(W(f))=e^{-(1 / 2) S(f, f)} .
$$

In the terminology of [4], the 2-point function $S$ of a quasifree state $\omega_{S}$ is a polarization of the phase space $(K, \Gamma, \gamma)$, i.e. a positive Hermitian form on $K$ such that

$$
S(f, g)-S(\Gamma g, \Gamma f)=\gamma(f, g) .
$$

Given $S$ one defines a scalar product $(\cdot, \cdot)_{S}$ on $K$ by

$$
(f, g)_{S}=S(f, g)+S(\Gamma g, \Gamma f)
$$

and we have $[4]$

$$
|S(f, g)|^{2} \leqq(f, f)_{S}(g, g)_{S},
$$

so that we have an operator $\tilde{S}$ defined on the Hilbert space completion $K_{S}$ of $K$ determined by $(\cdot, \cdot)_{S}$ and satisfying

$$
(f, \tilde{S} g)_{S}=S(f, g) .
$$

$\omega_{S}$ is a Fock state if and only if $\tilde{S}$ is a projection.

Araki and Yamagami [4] proved the following result:

Theorem 1.1. The GNS-representations of the Weyl algebra over a phase space $(K, \Gamma, \gamma)$ induced by quasifree states $\omega_{s}$ and $\omega_{s^{\prime}}$ are quasiequivalent if and only if the following conditions are satisfied:

(A1) $(\cdot, \cdot)_{S}$ and $(\cdot, \cdot)_{S^{\prime}}$ induce the same topology on $K$,

(A2) $\tilde{S}^{1 / 2}-\tilde{S}^{\prime 1 / 2} \in \mathscr{L}^{2}\left(K_{S}\right)$,

where $\mathscr{L}^{2}\left(K_{S}\right)$ denotes the set of Hilbert-Schmidt operators on $K_{S}$ and $\tilde{S}^{\prime}$ is the operator on $K_{S}$ representing the polarization $S^{\prime}$,

$$
\left(f, \tilde{S}^{\prime} g\right)_{S}=S^{\prime}(f, g) \text {. }
$$


Of course, when $\omega_{S}$ and $\omega_{S^{\prime}}$ are pure states and hence Fock states, quasiequivalence implies unitary equivalence. The above result will be applied in Sect. 4 not only to the adiabatic vacuum states themselves in the case of a RobertsonWalker spacetime with spherical spatial sections but also in the case of open Robertson-Walker spacetimes to the restrictions of the adiabatic vacuum states to the local algebras $\mathfrak{U}(\mathcal{O}), \mathcal{O} \in \mathscr{K}$ which are also quasifree states over a Weyl algebra.

Now if we take a state $\omega$ and restrict it to a state $\omega\lceil\mathcal{O}$ of $\mathfrak{U}(\mathcal{O})$ and then apply the GNS-construction we obtain a representation $\pi_{\omega \uparrow \mathcal{O}}$ which is a subrepresentation of $\pi_{\omega} \uparrow \mathcal{O}$. Hence the above theorem, when applicable, does not automatically lead to local quasiequivalence. We therefore complete the proof of local quasiequivalence in Sect. 5 by proving that $\pi_{\omega} \uparrow \mathcal{O}$ is factorial and hence quasiequivalent to $\pi_{\omega \mid \mathcal{O}}$.

We also prove in Sect. 5 that duality holds in the Fock representation $\pi_{\omega}$ constructed from an adiabatic vacuum state $\omega$. In Minkowski space duality has had important consequences for the superselection structure of the theory and it is gratifying to see that this property holds in curved space time, too.

The conclusions of this paper are that, for Robertson-Walker spacetimes, the concept of adiabatic vacuum states leads to a class of states compatible with the principle of local definiteness. We therefore propose, as a necessary condition, that any physical state of the free Klein-Gordon field on such spacetimes should be locally quasiequivalent to an adiabatic vacuum state. We comment briefly on the relation of the adiabatic vacuum states to the Hadamard form. Najmi and Ottewill [5] show that a Hadamard vacuum state $\omega_{s}$ for the Klein-Gordon field over a Robertson-Walker spacetime with flat spatial section, i.e. a Fock state with anticommutator function $(\cdot,)_{S}$ (cf. (1.9)) of the Hadamard form, is, in their terminology, an adiabatic vacuum state of order $\mathbf{t w o}^{2}$. Hence, we would get the same class of states compatible with the principle of local definiteness if we insisted on the Hadamard form. Bernard [6] considers Hadamard vacuum states over a Bianchi type I spacetime ${ }^{3}$ and a computation again shows that the high energy behaviour of such a state agrees with that of an adiabatic vacuum state of order two ${ }^{4}$. Further computations relating the Hadamard form and the adiabatic vacuum can be found in [7]. Thus we tentatively conclude that the approach using adiabatic vacuum states and the approach using Hadamard vacuum states are equivalent and viable methods for fixing the local states as required by the principle of local definiteness. It should be noted that the problem of renormalizing the energymomentum tensor, which motivatives the Hadamard form [5], is well posed once the local states are determined.

Although the adiabatic vacuum states and the Hadamard vacuum states seem to be equivalent methods of fixing the local states, the approach here using adiabatic vacuum states has some definite advantages. One needs a definition of the Hadamard condition which avoids convergence problems but guarantees the symmetry condition as well as the positivity condition (cf. (1.9)). Fulling et al. [8] give a definition of the Hadamard condition which meets these requirements. They

\footnotetext{
2 This corresponds to a single iteration in the terminology of Sect. 3

3 There are no obvious obstacles to extending our analysis to this class of spacetimes

4 It must be pointed out that (2.8) of [6] does not imply unitary equivalence of the Fock representations (cf. Sect. 4). It is conceivable that it implies local quasiequivalence, cf. the discussion in Sect. 3
} 
also show that there is a large class of Hadamard states for an arbitrary globally hyperbolic manifold. It nevertheless remains difficult to exhibit an explicit example of a Hadamard vacuum state for a general Robertson-Walker spacetime. Furthermore, even if one just wishes to fix the terms as far as is needed to guarantee local quasiequivalence, the necessary computations are more easily performed using the adiabatic states.

\section{Homogeneous Isotropic Quasifree States}

In this paper, we consider only homogeneous isotropic spacetimes where it is natural to try and specify the local quasiequivalence class of physical states by singling out a special class of homogeneous isotropic quasifree states. This section is devoted to describing the structure of the homogeneous isotropic quasifree states and showing that their 2-point functions depend only on the magnitude of the "3-momentum." We also characterize the homogeneous isotropic Fock states and show that any such state can be obtained in the usual fashion using separated mode solutions of the Klein-Gordon equation. Although these results are hardly surprising there does not appear to be any proof given in the literature. Readers not interested in the proof of these results might turn straight to the final result to familiarize themselves with the notation and pass on to Sect. 3 .

The homogeneous isotropic spacetimes have the form $\mathscr{M}=\mathbb{R} \times \mathscr{S}$, where $\mathscr{S}$ is a homogeneous Riemannian manifold and with respect to this decomposition the metric takes on the Robertson-Walker form:

$$
d s^{2}=d t^{2}-a(t)^{2} h_{i j}(y) d y^{i} d y^{j}
$$

where $h$ is the metric on $\mathscr{S}$. We will assume that $a$ is a strictly positive smooth function. There are three possibilities for $\mathscr{S}$ corresponding to constant positive, zero and negative curvature which we denote by $\mathscr{S}^{\varepsilon}, \varepsilon \in\{+, 0,-\}$ and it is convenient to regard $\mathscr{S}^{\varepsilon}$ as being embedded in $\mathbb{R}^{4}$ :

$$
\begin{aligned}
\mathscr{S}^{+} & =\left\{x \in \mathbb{R}^{4}: x^{0^{2}}+\sum_{i=1}^{3} x^{i^{2}}=1\right\}, \\
\mathscr{S}^{0} & =\left\{x \in \mathbb{R}^{4}: x^{0}=0\right\}, \\
\mathscr{S}^{-} & =\left\{x \in \mathbb{R}^{4}: x^{0^{2}}-\sum_{i=1}^{3} x^{i^{2}}=1, x^{0}>0\right\} .
\end{aligned}
$$

The metric $h^{\varepsilon}$ is that induced on $\mathscr{S}^{\varepsilon}$ by the Euclidean metric on $\mathbb{R}^{4}$ for $\varepsilon=+, 0$ and the Minkowski metric for $\varepsilon=-$. These spaces are homogeneous spaces for groups $G^{\varepsilon}$, which are the rotation group $S O(4)$, the Euclidean group $E(3)$ and the Lorentz group $\mathscr{L}_{+}^{\uparrow}(4)$ respectively. The group $G^{\varepsilon}$ also acts as a group of isometries of the manifold $\mathscr{M}^{\varepsilon}:=\mathbb{R} \times \mathscr{S}^{\varepsilon}, g(t, x)=(t, g x)$. In what follows we will omit the symbol $\varepsilon$ unless it is necessary to specify one of its values.

Now the mapping $E: \mathscr{D}(\mathscr{M}) \rightarrow C^{\infty}(\mathscr{M})$, defined in Sect. 1, commutes with the natural action of $G$ being a group of isometries so that $G$ acts as a group of Bogoliubov transformations of the phase space $(K, \Gamma, \gamma)$ and hence induces a group of Bogoliubov automorphisms of the self-dual CCR-algebra and of the Weyl 
algebra:

$$
\alpha_{g}(\varphi(f))=\varphi(g f), \quad \alpha_{g}(W(f))=W(g f), \quad g \in G .
$$

A state $\omega$ is said to be homogeneous and isotropic if $\omega \circ \alpha_{g}=\omega, g \in G$. Since a quasifree state $\omega_{S}$ is uniquely determined by its 2-point function $S$, it will be homogeneous and isotropic if and only if

$$
S\left(g f, g f^{\prime}\right)=S\left(f, f^{\prime}\right), \quad g \in G .
$$

To be able to analyse this condition effectively some continuity conditions on the 2-point function $S$ are necessary and indeed physically desirable. It would be natural for example to demand that $S$ be separately continuous in $f$ and $f^{\prime}$ relative to the usual topology on $\mathscr{D}(\mathscr{M})$. We shall impose a stronger, but still rather weak, continuity condition. To formulate this, we first need to describe our phase space $(K, \Gamma, \gamma)$ in terms of Cauchy data on a Cauchy surface $\mathscr{S}_{t}:=t \times \mathscr{S}$.

A smooth 3-manifold such as $\mathscr{S}$ has a phase space $(L, \Gamma, \gamma)$ corresponding to the canonical commutation relations. An element of $L$ is a pair $F=(f, h)$, where $f \in \mathscr{D}(\mathscr{S})$, the set of complex-valued odd smooth 3-forms with compact support and $h \in C_{0}^{\infty}(\mathscr{S})$, the set of complex-valued smooth functions with compact support. $\Gamma$ corresponds to complex conjugation whilst the Hermitian form $\gamma$ is given by

$$
\gamma\left(F, F^{\prime}\right)=i \int\left(\bar{f} \wedge h^{\prime}-\bar{h} \wedge f^{\prime}\right) .
$$

We now let $j_{t}: \mathscr{S} \rightarrow \mathscr{M}$ denote the (oriented) embedding mapping $j_{t}(y)=(t, y)$, then we have an isomorphism of phase spaces $\rho_{t}:(K, \Gamma, \gamma) \rightarrow(L, \Gamma, \gamma)$, defined by

$$
\rho_{t}(f):=\left(j_{t}^{*} \# d E f,-j_{t}^{*} E f\right),
$$

where $d$ denotes the exterior derivative. This isomorphism describes the passage from the field $\varphi$ on $\mathscr{M}$ to its Cauchy data on the Cauchy surface $\mathscr{S}_{t}$ in terms of test functions. It is clearly compatible with the natural action of $G$ so we may use $(L, \Gamma, \gamma)$ to investigate the structure of the homogeneous isotropic quasifree states.

We now describe the continuity condition imposed on the 2-point function $S$. By virtue of the Riemannian metric on $\mathscr{S}$ we have a scalar product on $L$,

$$
\left(F, F^{\prime}\right):=\int\left(\bar{f} \wedge \# f^{\prime}+\bar{h} \wedge \# h^{\prime}\right)
$$

We let the Laplacian $\Delta$ act componentwise and give $L$ the topology defined by the norms $\|\cdot\|_{v}$, where

$$
\|F\|_{v}^{2}:=\left(F,\left(-\Delta+m^{2}\right)^{2 v} F\right), \quad v \in \mathbb{N}_{0},
$$

and require $S: L \times L \rightarrow \mathbb{C}$ to be jointly continuous with respect to this topology. Thus there is an integer $v$ and a constant $C>0$ such that

$$
\left|S\left(F, F^{\prime}\right)\right| \leqq C\|F\|_{v}\left\|F^{\prime}\right\|_{v} .
$$

But this means by the Riesz Representation Theorem that there is a bounded operator $\hat{S}$ on the Hilbert space $H_{v}$ obtained by completing $L$ in the norm $\|\cdot\|_{v}$ such that

$$
S\left(F, F^{\prime}\right)=\left(F, \hat{S} F^{\prime}\right)_{v}
$$

The natural action of $G$ on $L$ is isometric for each of the norms $\|\cdot\|_{\nu}$ and thus extends to a unitary representation in each $H_{v}$ so that $S$ is invariant under $G$ if 
and only if $\hat{S}$ is in the commutant of this representation. There is a unitary mapping $V$ from $H_{v}$ to $L^{2}(\mathscr{S}) \oplus L^{2}(\mathscr{S})$ defined by

$$
V(F)=\#\left(-\Delta+m^{2}\right)^{v} f \oplus\left(-\Delta+m^{2}\right)^{v} h, \quad F \in L,
$$

since $\left(-\Delta+m^{2}\right)^{v}$ is essentially self-adjoint on $C_{0}^{\infty}(\mathscr{S})$ and strictly positive, $v \in \mathbb{N}_{0}$ [9]. Here $L^{2}(\mathscr{S})$ is, of course, defined using the invariant measure derived from the Riemannian metric on $\mathscr{S} . V$ intertwines the representation of $G$ on $H_{v}$ with the unitary representation $U \oplus U$ on $L^{2}(\mathscr{S}) \oplus L^{2}(\mathscr{S})$ given by

$$
U(g)(h)=h \circ g^{-1}, \quad h \in \mathscr{C}_{0}(\mathscr{S}) .
$$

Hence we shall be able to analyse the $G$-invariance of the 2-point functions by computing the commutant of $U$.

The representation theory of $G$ is treated in $[10,11]$ and the relevant facts are summarized in an appendix. The representation $U$, known as the quasiregular representation, is decomposed into irreducible representations by what amounts to a Fourier transformation. The simplest case is that of $G^{+}=S O(4)$, where the eigenspaces of the Laplace operator $\Delta$ give a decomposition into a direct sum of inequivalent irreducible representations. If $\vec{k}=(k, l, m)$, where $k \in \mathbb{N}_{0}, l=0,1, \ldots, k$, $m=-l,-l+1, \ldots, l$, then there is a conventional orthonormal basis $\mathscr{Y}_{\vec{k}}$ of eigenvectors of $\Delta$ in $L^{2}\left(\mathscr{S}^{+}\right)$,

$$
\Delta \mathscr{Y}_{\vec{k}}=-k(k+2) \mathscr{Y}_{\vec{k}},
$$

and the Fourier transformation

$$
\tilde{h}(\vec{k}):=\left(\mathscr{Y}_{\vec{k}}, h\right)
$$

gives a unitary transformation of $L^{2}\left(\mathscr{S}^{+}\right)$onto $L^{2}\left(\tilde{\mathscr{S}}^{+}\right)$, where $\tilde{\mathscr{S}}^{+}$denotes the momentum space associated with $\mathscr{S}^{+}$, i.e. the range of values of $\vec{k}$ equipped with the counting measure. A bounded operator on $L^{2}\left(\mathscr{S}^{+}\right)$commuting with $U$ corresponds to multiplication by a bounded function of $k$ on $L^{2}\left(\tilde{\mathscr{S}}^{+}\right)$.

The case $G^{0}=E(3)$ is very familiar since the representation $U$ is decomposed into a direct integral of irreducible representations using the usual Fourier transform. The Laplace operator now has no eigenvectors in $L^{2}\left(\mathscr{S}^{0}\right)$ but we use the generalized eigenvectors

$$
\mathscr{Y}_{\vec{k}}(\vec{x}):=(2 \pi)^{3 / 2} e^{i \vec{k} \cdot \vec{x}}, \quad \Delta \mathscr{Y}_{\vec{k}}=-k^{2} \mathscr{Y}_{\vec{k}},
$$

which we treat as generalized functions on $\mathscr{S}^{0}$. The Fourier transform

$$
\tilde{h}(\vec{k}):=\left(\mathscr{Y}_{\vec{k}}, h\right), \quad h \in C_{0}^{\infty}\left(\mathscr{S}^{0}\right),
$$

extends to a unitary operator from $L^{2}\left(\mathscr{S}^{0}\right)$ to $L^{2}\left(\tilde{\mathscr{S}}^{0}\right)$. A bounded operator on $L^{2}\left(\mathscr{S}^{0}\right)$ commuting with the translation subgroup of $G^{0}$ corresponds to multiplication by an essentially bounded measurable function of $\vec{k}$ in $L^{2}\left(\widetilde{\mathscr{S}}^{0}\right)$. Hence a bounded operator commuting with $U$ will correspond to multiplication by an essentially bounded measurable function of $k=|\vec{k}|$.

The case $G^{-}=\mathscr{L}_{+}^{\dagger}(4)$ is similar since the representation $U$ is decomposed into a direct integral of irreducible representations using a suitable modification of the Fourier transform. We again use the generalized eigenvectors of the Laplace operator

$$
\mathscr{Y}_{\vec{k}}(x)=(2 \pi)^{-3 / 2}(x \cdot \xi)^{-1+i k}, \quad \vec{k}=k \vec{\xi} \in \mathbb{R}^{3}, \quad k=|\vec{k}|, \quad \Delta \mathscr{Y}_{\vec{k}}=-\left(k^{2}+1\right) \mathscr{Y}_{\vec{k}} .
$$


Here $\mathscr{S}^{-}$is regarded as being embedded in $\mathbb{R}^{4}, \xi=(1, \vec{\xi}) \in \mathbb{R}^{4}$ and $x \cdot \xi$ refers to the Minkowski scalar product. Regarding $\mathscr{Y}_{\vec{k}}$ as a generalized function we have an analogue of the Fourier transform defined by

$$
\tilde{h}(\vec{k}):=\left(\mathscr{Y}_{\vec{k}}, h\right), \quad h \in C_{0}^{\infty}\left(\mathscr{S}^{-}\right),
$$

and extending to a unitary operator from $L^{2}\left(\mathscr{S}^{-}\right)$to $L^{2}(\tilde{\mathscr{S}}-):=L^{2}\left(\mathbb{R}^{3}\right)$. A bounded operator commuting with the representation $U$ again corresponds to multiplication with an essentially bounded measurable function of $k=|\vec{k}|$. Our proof of this result is relatively technical since it relies on [12; Proposition 8.6.4] and we have relegated it to the appendix.

To have a uniform notation for the eigenvalues of the Laplacian we write

$$
\Delta \mathscr{Y}_{\vec{k}}=-E(k) \mathscr{Y}_{\vec{k}}
$$

in each of the above three cases.

From the above computation of the commutant of $U$ and the fact that the Fourier transform of the Laplace operator is just multiplication by a function of $k$, we see that the 2-point function $S$ of a homogeneous isotropic quasifree state satisfying the continuity condition (2.11) has the following form:

$$
S\left(F, F^{\prime}\right)=\int d \vec{k}\left\langle\tilde{F}(\vec{k}), S(k) \tilde{F}^{\prime}(\vec{k})\right\rangle, \quad F, F^{\prime} \in L,
$$

where

$$
\left\langle\tilde{F}(\vec{k}), S(k) \tilde{F}^{\prime}(\vec{k})\right\rangle=\sum_{i, j=0}^{1} \overline{\tilde{F}_{i}(\vec{k})} S_{i j}(k) \tilde{F}_{j}^{\prime}(\vec{k}),
$$

$k \mapsto S_{i j}(k)$ is measurable and polynomially bounded and if $F=(f, h) \in L$ then

$$
\tilde{F}_{0}(\vec{k})=\overparen{\# f}(\vec{k}) \text { and } \tilde{F}_{1}(\vec{k})=\tilde{h}(\vec{k}) .
$$

Of course, when $\varepsilon=+$ then $\int \overrightarrow{d k}$ is just a summation over $\vec{k}$ and $S_{i j}$ is automatically measurable.

Now to be the 2-point function of a quasifree state $S$ must be a polarization of the phase space $(L, \Gamma, \gamma)$. It is thus a positive Hermitian form satisfying the analogue of (1.8). Using the unitarity of the Fourier transform and Eq. (A.7) we have

$$
\begin{aligned}
\gamma\left(F, F^{\prime}\right) & =\int d \vec{k}\left\langle\tilde{F}(\vec{k}), \hat{\gamma} \tilde{F}^{\prime}(\vec{k})\right\rangle, \\
S\left(\Gamma F^{\prime}, \Gamma F\right) & =\int d \vec{k}\left\langle\tilde{F}(\vec{k}), S^{t}(k) \tilde{F}^{\prime}(\vec{k})\right\rangle,
\end{aligned}
$$

where $S^{t}(k)$ is the transposed matrix and $\hat{\gamma}:=\left(\begin{array}{cc}0 & i \\ -i & 0\end{array}\right)$. Thus we get conditions
on the entries of $S(k)$. Lemma 2.1. The Hermitian form $S(\cdot, \cdot)$ defined by (2.22) is a polarization if and only if the following conditions on the entries $S_{i j}(k)$ of the matrix $S(k)$ hold almost everywhere (a.e.) in $k$ :
a)$$
S_{01}(k)-S_{10}(k)=i
$$

$$
S_{01}(k)=\overline{S_{10}(k)} ; \quad S_{00}(k) \geqq 0 ; \quad S_{00}(k) S_{11}(k)-\left|S_{01}(k)\right|^{2} \geqq 0 .
$$

Proof. For technical reasons it is advantageous to write $S(\cdot, \cdot)$ in the form

$$
S\left(F, F^{\prime}\right)=\left(\tilde{V} F, T \tilde{V} F^{\prime}\right), \quad F, F^{\prime} \in L,
$$

where $\tilde{V}: H_{v} \rightarrow L^{2}(\tilde{\mathscr{S}}) \oplus L^{2}(\tilde{\mathscr{S}})$ denotes the unitary operator arising from the 
composition of $V$ of $(2.13)$ with the Fourier transformation. $T \in \mathscr{B}\left(L^{2}(\tilde{\mathscr{S}}) \oplus L^{2}(\tilde{\mathscr{S}})\right)$ corresponds to multiplication by a matrix $T(k)$ whose entries $T_{i j}(k)$ are measurable, essentially bounded and uniquely determined up to a set of measure zero. The $T_{i j}(k)$ are related to the entries of $S(k)$ by

$$
S_{i j}(k)=\left(m^{2}+E(k)\right)^{2 v} T_{i j}(k), \quad \text { a.e.. }
$$

It is now clear that (1.8) is equivalent to $(2.27)$. Since $S(\cdot, \cdot)$ is positive, $T$ must be a positive operator and hence the square of its positive square root. Thus, bearing (2.30) in mind, we see that the positivity of $S(\cdot, \cdot)$ is equivalent to (2.28).

The next lemma characterizes the Fock states where the operator $\tilde{S}$ of (1.11) is a projection.

Lemma 2.2. A homogeneous, isotropic, quasifree state $\omega_{s}$ is a Fock state if and only if

$$
S_{00}(k) S_{11}(k)-\left|S_{01}(k)\right|^{2}=0, \quad \text { a.e. }
$$

Proof. It follows from (1.9), (2.26) and (2.30) that

$$
\left(F, F^{\prime}\right)_{S}=\left(\tilde{V} F,\left(T+T^{t}\right) \tilde{V} F^{\prime}\right), \quad F, F^{\prime} \in L,
$$

where $T^{t}$ is defined by multiplication with the transpose of the matrix $T(k)$. Let $W$ be the unique positive square root of $T+T^{t} . W$.is defined by the matrix $W(k)=\left[T(k)+T^{t}(k)\right]^{1 / 2}$ whose entries $W_{i j}(k)$ are essentially bounded, measurable, real functions and $W=W^{t}$. Let

$$
\Delta(k):=\operatorname{det} W(k)=\left[\operatorname{det}\left(T(k)+T^{t}(k)\right)\right]^{1 / 2},
$$

then, since by (2.27), (2.28) and (2.30) $\operatorname{Im} T_{01}(k)=\frac{1}{2}\left(m^{2}+E(k)\right)^{-2 v}$ a.e.,

$$
\Delta(k)=2\left[T_{00}(k) T_{11}(k)-\left(\operatorname{Re} T_{01}(k)\right)^{2}\right]^{1 / 2}=2\left[\operatorname{det} T(k)+\left(\operatorname{Im} T_{01}(k)\right)^{2}\right]^{1 / 2}>0 \quad \text { a.e.. }
$$

Thus $W$ has an inverse $W^{-1}$ given by the matrix

$$
W^{-1}(k)=\frac{1}{\Delta(k)}\left(\begin{array}{rr}
W_{11}(k) & -W_{01}(k) \\
-W_{01}(k) & W_{00}(k)
\end{array}\right)
$$

whose entries are measurable and essentially polynomially bounded. If we write

$$
T(k)=\frac{1}{2}\left(T(k)+T^{t}(k)\right)+\operatorname{Im} T_{01}(k)\left(\begin{array}{rr}
0 & i \\
-i & 0
\end{array}\right)
$$

then a computation shows that $W^{-1} T W^{-1}$ is given by multiplication with the matrix

$$
\frac{1}{2}\left(\begin{array}{ll}
1 & 0 \\
0 & 1
\end{array}\right)+\frac{\operatorname{Im} T_{01}(k)}{\Delta(k)}\left(\begin{array}{rr}
0 & i \\
-i & 0
\end{array}\right)
$$

and, since by (2.33)

$$
\left|\frac{\operatorname{Im} T_{01}(k)}{\Delta(k)}\right| \leqq \frac{1}{2} \text { a.e., }
$$

$W^{-1} T W^{-1}$ extends to a bounded operator which is a projection if and only if 
equality holds in (2.34), i.e. by (2.33) if and only if

$$
T_{00}(k) T_{11}(k)-\left|T_{01}(k)\right|^{2}=0 \quad \text { a.e.. }
$$

Since, by (2.30), this is equivalent to (2.31) we may complete the proof by showing that the bounded operator extending $W^{-1} T W^{-1}$ is unitarily equivalent to $\tilde{S}$. To this end, we note that there is a unique isometry $U: L_{S} \rightarrow L^{2}(\tilde{\mathscr{S}}) \oplus L^{2}(\tilde{\mathscr{S}})$ defined by

$$
U F=W \tilde{V} F, \quad F \in L .
$$

Further $U L$ is dense in $L^{2}(\tilde{\mathscr{S}}) \oplus L^{2}(\tilde{\mathscr{S}})$ since $(\tilde{G}, U F)=0, F \in L$, implies $(W \widetilde{G}, \tilde{V} F)=0$, $F \in L$, and hence $\widetilde{G}=W^{-1} W \widetilde{G}=0$. Thus $U$ is unitary and

$$
\left(U F, U \tilde{S} U^{-1} U F^{\prime}\right)=\left(F, \tilde{S} F^{\prime}\right)_{S}=S\left(F, F^{\prime}\right)=\left(\tilde{V} F, T \tilde{V} F^{\prime}\right)=\left(U F, W^{-1} T W^{-1} U F^{\prime}\right)
$$

completing the proof.

With the help of Lemmas 2.1 and 2.2, we may summarize our results in the following theorem.

Theorem 2.3. The homogeneous, isotropic Fock states for the free Klein-Gordon field in a Robertson-Walker spacetime satisfying the continuity condition (2.11) are given by 2-point functions of the form (2.22). The entries of the matrix $S(k)$ can be expressed in the form

$$
S_{00}(k)=|q(k)|^{2}, \quad S_{11}(k)=|p(k)|^{2}, \quad S_{01}(k)=\overline{q(k)} p(k),
$$

where $p$ and $q$ are essentially polynomially bounded measurable functions satisfying

$$
\overline{q(k)} p(k)-q(k) \overline{p(k)}=i .
$$

Conversely, every pair of essentially polynomially bounded measurable functions satisfying (2.36) yields via (2.35) and (2.22) the 2-point function of a homogeneous, isotropic Fock state satisfying the continuity condition (2.11).

Proof. Using the notation of Lemmas 2.1 and 2.2, we see that $T_{01}(k) \neq 0$ a.e. and we define a bounded measurable function $\varphi$ by setting

$$
\varphi(k)=1, \quad \text { if } \quad T_{01}(k)=0 \quad \text { and } \quad \varphi(k)=\frac{T_{01}(k)}{\left|T_{01}(k)\right|} \text { otherwise }
$$

We now set, for example,

$$
Q(k):=\left(T_{00}(k)\right)^{1 / 2}, \quad P(k)=\left(T_{11}(k)\right)^{1 / 2} \varphi(k),
$$

then $Q$ and $P$ are measurable and essentially bounded and we get from (2.31)

$$
\overline{Q(k)} P(k)=T_{01}(k) \frac{\left(T_{00}(k) T_{11}(k)\right)^{1 / 2}}{\left|T_{01}(k)\right|}=T_{01}(k) \quad \text { a.e. }
$$

so that

$$
\overline{Q(k)} P(k)-Q(k) \overline{P(k)}=2 i \operatorname{Im} T_{01}(k)=i\left(m^{2}+E(k)\right)^{-2 v} .
$$

Thus, by (2.30), if we set $q(k):=\left(m^{2}+E(k)\right)^{v} Q(k)$ and $p(k):=\left(m^{2}+E(k)\right)^{v} P(k)$, we obtain functions with the desired properties. Conversely, given $p$ and $q$ as in the statement of the theorem, then for $v$ sufficiently large the matrix $T(k)$ given by 
(2.35) and (2.30) defines a bounded operator and we see from (2.22) that the 2-point function $S(\cdot, \cdot)$ satisfies the required continuity condition (2.11).

Remark. If we replace $q(k)$ and $p(k)$ by $e^{i \psi(k)} q(k)$ and $e^{i \psi(k)} p(k)$, where, $\psi(k)$ is a real measurable function, we obtain the same 2-point function and hence the same quasifree state.

With the help of $p$ and $q$, we may realize the quasifree Fock state with 2-point function $S(\cdot, \cdot)$ on a Bosonic Fock space with 1-particle space $L^{2}(\tilde{\mathscr{S}})$ and annihilation and creation operators $a$ and $a^{*}$,

$$
\left[a(\tilde{h}), a^{*}\left(\tilde{h}^{\prime}\right)\right]=\left(\tilde{h}, \tilde{h}^{\prime}\right), \quad \tilde{h}, \tilde{h}^{\prime} \in L^{2}(\tilde{\mathscr{S}}) .
$$

In terms of a field $\vartheta$ on the Cauchy surface $\mathscr{S}_{t}$ and its canonical conjugate momentum $\pi$, we take

$$
\vartheta_{S}(f)=a(q \widetilde{\# \tilde{f}})+a^{*}(q \widetilde{\# f}) ; \quad \pi_{S}(h)=a(p \tilde{\bar{h}})+a^{*}(p \tilde{h}) ; \quad F=(f, h) \in L .
$$

From this, we get, as in [3], the corresponding representation of the field $\varphi$ by setting

$$
\varphi_{s}(f)=\vartheta_{s}\left(j_{t}^{*} \# d E f\right)-\pi_{s}\left(j_{t}^{*} E f\right), \quad f \in \mathscr{D}(\mathscr{M}) .
$$

In order to express this representation of the field in the more usual fashion in terms of mode solutions of the Klein-Gordon equation, we give an explicit expression for the operator $E$ in terms of such modes. An expression of the form $T_{k}\left(x^{0}\right) \mathscr{Y}_{\vec{k}}(\vec{x})$ will be a solution of the Klein-Gordon equation if $T_{k}$ satisfies the differential equation

$$
\ddot{T}_{k}+3 \frac{\dot{a}}{a} \dot{T}_{k}+\omega_{k}^{2} T_{k}=0 ; \quad \omega_{k}^{2}:=\frac{E(k)}{a^{2}}+m^{2} .
$$

We demand in addition that $T_{k}$ satisfy, at time $t$, the equation

$$
\bar{T}_{k} \dot{T}_{k}-\dot{\bar{T}}_{k} T_{k}=i a^{-3}
$$

Since $T_{k}$ satisfies $(2.40),(2.41)$ is then satisfied at all times.

We now define a generalized function by

$$
\begin{aligned}
G(x, y) & :=\int d \vec{k} G_{k}\left(x^{0}, y^{0}\right) \mathscr{Y}_{\vec{k}}(\vec{x}) \overline{\mathscr{Y}_{\vec{k}}(\vec{y})}, \\
G_{k}\left(x^{0}, y^{0}\right) & :=i\left(T_{k}\left(x^{0}\right) \bar{T}_{k}\left(y^{0}\right)-\bar{T}_{k}\left(x^{0}\right) T_{k}\left(y^{0}\right)\right),
\end{aligned}
$$

and use it to define an operator $E^{\prime}: \mathscr{D}(\mathscr{M}) \rightarrow C^{\infty}(\mathscr{M})$ :

$$
\begin{aligned}
\left(E^{\prime} f\right)(x) & :=-G(x, f):=\int d y^{0} \int d \vec{k} \mathscr{Y}_{\vec{k}}(\vec{x}) G_{k}\left(x^{0}, y^{0}\right) \check{f}\left(y^{0}, \vec{k}\right), \\
\check{f}(t, \vec{k}): & =a^{3}(t)\left(\mathscr{Y}_{\vec{k}}, j_{t}^{*} \# f\right) .
\end{aligned}
$$

Remark. $G_{k}\left(\cdot, y^{0}\right)$ satisfies, for each fixed $y^{0}$, the differential equation (2.40) with initial conditions $G_{k}\left(y^{0}, y^{0}\right)=0, \dot{G}_{k}\left(y^{0}, y^{0}\right)=-a^{-3}\left(y^{0}\right)$. $G_{k}$ is therefore independent of the particular solution $T_{k}$ chosen. (2.39).

The following lemma allows us to prove that $E^{\prime}$ agrees with the operator $E$ of

Lemma 2.4. Let $f \in \mathscr{D}(\mathscr{M})$ then $E^{\prime} f$ satisfies the Klein-Gordon equation. If $u$ is a solution of the Klein-Gordon equation whose initial data $u_{0}, u_{1}$ are $C^{\infty}$ with compact 
support,

$$
u_{0}=j_{t}^{*} u, \quad u_{1}=j_{t}^{*} \# d u,
$$

then

$$
\int_{\mathscr{M}} u \wedge f=\int_{\mathscr{S}}\left(u_{0} \wedge j_{t}^{*}\left(\# d E^{\prime} f\right)-u_{1} \wedge j_{t}^{*} E^{\prime} f\right) .
$$

Proof. Differentiating under the integral sign in (2.44) using (2.21) and the fact that the $T_{k}$ satisfy (2.40), leads to $\left(\square+m^{2}\right) E^{\prime} f=0$. The justification for this step involves properties of the solutions of (2.40) and of the Fourier transform and will be relegated to Appendix C. Using (2.44), (2.45) and (A.7) we conclude that

$$
\begin{aligned}
& \int_{\mathscr{S}}\left(u_{0} \wedge j_{t}^{*}\left(\# d E^{\prime} f\right)-u_{1} \wedge j_{t}^{*} E^{\prime} f\right) \\
& \quad=\int d y^{0} \int d \vec{k}\left\{\tilde{u}_{0}(\vec{k}) a^{3}(t) \frac{\partial G_{k}}{\partial t}\left(t, y^{0}\right)-\widetilde{\# u_{1}}(\vec{k}) G_{k}\left(t, y^{0}\right)\right\} \overline{\tilde{f}}\left(y^{0}, \vec{k}\right) \\
& \quad=-\int d y^{0} d \mu(\vec{y}) a^{3}\left(y^{0}\right)(\# f)(y) u(y)=\int f \wedge u .
\end{aligned}
$$

Here $\mu(\vec{y})$ denotes the measure derived from the Riemannian metric on $\mathscr{S}$ and

$$
u(y)=-\int d \vec{k} \mathscr{Y}_{\vec{k}}(\vec{y})\left\{\tilde{u}_{0}(\vec{k}) a^{3}(t) \frac{\partial G_{k}}{\partial t}\left(t, y^{0}\right)-\widetilde{\# u_{1}}(\vec{k}) G_{k}\left(t, y^{0}\right)\right\}
$$

is the (unique) solution of the Klein-Gordon equation with (2.46) as initial conditions. This can be seen by differentiating the integral sign and will be justified in Appendix C.

Now $E$ also satisfies (2.47) (cf. [3; Lemma A.1]) so

$$
j_{t}^{*} \# d\left(E-E^{\prime}\right) f=0, \quad j_{t}^{*}\left(E-E^{\prime}\right) f=0, \quad f \in \mathscr{D}(\mathscr{M}) .
$$

But the solutions of the Klein-Gordon equation are uniquely determined by their initial data and we conclude that $E=E^{\prime}$.

In order to express the representation $\varphi_{S}$ of (2.39) in terms of mode solutions of the Klein-Gordon equation, we take initial conditions for $T_{k}$ at time $t$ given by

$$
T_{k}(t)=q(k) ; \quad \dot{T}_{k}(t)=a^{-3}(t) p(k) .
$$

We can now compute the test functions appearing in the expansion of $\varphi_{S}$ in terms of annihilation and creation operators using (2.38) and (2.39). Using (2.44) and the Fourier inversion formula (A.8) we find that the Fourier transform of \# $j_{t}^{*} \# d E f$ is $a^{3}(t) \int d y^{0} \dot{G}_{k}\left(t, y^{0}\right) \check{f}\left(y^{0}, \vec{k}\right)$ and of $j_{t}^{*} E f$ is $\int d y^{0} G_{k}\left(t, y^{0}\right) \check{f}\left(y^{0}, \vec{k}\right)$. Now

$$
\begin{aligned}
\int d y^{0}\left\{q(k) a^{3}(t) \dot{G}_{k}\left(t, y^{0}\right)-p(k) G_{k}\left(t, y^{0}\right)\right\} \check{f}\left(y^{0}, \vec{k}\right) & =-\int d y^{0} T_{k}\left(y^{0}\right) \check{f}\left(y^{0}, \vec{k}\right) \\
& =\int T_{k}\left(y^{0}\right) \mathscr{Y}_{\vec{k}}(\vec{y}) \wedge f(y) .
\end{aligned}
$$

Thus $\varphi_{S}(f)=\int \varphi_{S}(x) \wedge f(x)$, where

$$
\varphi_{S}(x)=\int d \vec{k}\left\{\bar{T}_{k}\left(x^{0}\right) \mathscr{Y}_{\vec{k}}(\vec{x}) a(\vec{k})+T_{k}\left(x^{0}\right) \overline{\mathscr{Y}_{\vec{k}}(\vec{x})} a(k)^{*}\right\}
$$

is the desired expression for the field in terms of mode solutions of the Klein-Gordon equation.

Conversely, starting from (2.49) we may define a representation of the field $\vartheta$ 
on a Cauchy surface and its canonical conjugate momenta $\pi$ by setting

$$
\vartheta_{S}(f)=\int j_{t}^{*} \varphi_{S} \wedge f ; \quad \pi_{s}(h)=\int j_{t}^{*} \# d \varphi_{s} \wedge h ; \quad F=(f, h) \in L .
$$

We may summarize the contents of this section by saying that a homogeneous, isotropic Fock state satisfying the continuity condition (2.11) yields a representation of the Klein-Gordon field of the form (2.49). The representation can be characterised either by the choice of the solutions $T_{k}$ of the differential equation (2.40) satisfying the normalization condition (2.41) or in terms of the functions $q(k), p(k)$ which fix the initial conditions according to (2.48). In the next section we study a special class of homogeneous isotropic Fock states, the adiabatic vacuum states.

\section{Adiabatic Vacuum States}

The concept of adiabatic vacuum state was first introduced by Parker [13]. It was designed to provide an optimal definition of "physical particle" in an expanding universe, optimal in the sense that the rate of production of these particles caused by the expansion was to be minimal. Furthermore, in the limit where the rate of expansion tends to zero, the notion of particle should reduce to the usual notion in the corresponding static universe. We do not adopt this point of view and the concept of "physical particle" plays no role here. Instead we extract from the concept of an adiabatic vacuum a procedure for fixing the behaviour of our parameters $q(k)$ and $p(k)$ in Theorem 2.3 for large values of $k$ in such a way that the corresponding family of Fock states is consistent with the principle of local definiteness.

The starting point, which can be traced back to the work of Parker, is to make an Ansatz of the WKB-type for the time-dependent part of the mode solutions determining our Fock states. Thus we write

$$
T_{k}\left(x^{0}\right)=a^{-3 / 2}\left(x^{0}\right)\left(2 \Omega_{k}\left(x^{0}\right)\right)^{-1 / 2} \exp \left(i \int_{t_{0}}^{x^{0}} \Omega_{k}(t) d t\right),
$$

where the positive functions $\Omega_{k}$ have still to be determined. The $T_{k}$ automatically satisfy (2.41) and if they are to satisfy the differential equation (2.40) we must have

$$
\Omega_{k}^{2}=\omega_{k}^{2}-\frac{3}{4}\left(\frac{\dot{a}}{a}\right)^{2}-\frac{3}{2} \frac{\ddot{a}}{a}+\frac{3}{4}\left(\frac{\dot{\Omega}_{k}}{\Omega_{k}}\right)^{2}-\frac{1}{2} \frac{\ddot{\Omega}_{k}}{\Omega_{k}} .
$$

One could try to solve this equation iteratively:

$$
\begin{aligned}
\left(\Omega_{k}^{(n+1)}\right)^{2} & =\omega_{k}^{2}-\frac{3}{4}\left(\frac{\dot{a}}{a}\right)^{2}-\frac{3}{2} \frac{\ddot{a}}{a}+\frac{3}{4}\left(\frac{\dot{\Omega}_{k}^{(n)}}{\Omega_{k}^{(n)}}\right)^{2}-\frac{1}{2} \frac{\ddot{\Omega}_{k}^{(n)}}{\Omega_{k}^{(n)}} \\
\left(\dot{\Omega}_{k}^{(0)}\right)^{2} & =\omega_{k}^{2}=\frac{E(k)}{a^{2}}+m^{2} .
\end{aligned}
$$

Now, as $a$ is a completely arbitrary strictly positive smooth function, we can never rule out the possibility that there will be values of $k$ and $x^{0}$ where (3.3) yields a negative value for $\left(\Omega^{(n+1)}\right)^{2}$. The iteration procedure then breaks down so there is certainly no hope, in general, of obtaining a solution $T_{k}$ of (2.40), and hence a 
state by iteration. However, we only need a class of states and we will use (3.1), after a finite number of iterations, merely to determine suitable initial values for the solution of (2.40). Moreover, provided we limit ourselves to a finite interval $I \subset \mathbb{R}$ in time and $k$ is chosen sufficiently large, there are no difficulties with the iteration procedure. As will become clear shortly, the following result can be proved by induction on $n$.

Lemma 3.1. Let $I \subset \mathbb{R}$ be a closed interval, then, for each $n \in \mathbb{N}_{0}$ there is $a \varkappa_{n}(I) \geqq 0$ so that, setting

$$
R_{n}(I):=\left\{\left(k, x^{0}\right): x^{0} \in I, k \geqq \varkappa_{n}(I)\right\},
$$

$\Omega^{(n)}$ is strictly positive on $R_{n}(I)$ and $\Omega^{(n)}$ and all its derivatives with respect to time are continuous on $R_{n}(I)$. Furthermore, there are constants $A, B>0$ such that

$$
A(1+k) \leqq \Omega_{k}^{(n)}\left(x^{0}\right) \leqq B(1+k), \quad\left(k, x^{0}\right) \in R_{n}(I) .
$$

The $\Omega^{(n)}$ obtained by iteration in Lemma 3.1 can be continued to strictly positive functions on $\left\{\left(k, x^{0}\right): k \geqq 0, x^{0} \in I\right\}$ so as to be continuous together with all their derivatives with respect to time.

We now define for $t_{0}, t \in I$,

$$
W_{k}^{(n)}(t):=a^{-3 / 2}(t)\left(2 \Omega_{k}^{(n)}(t)\right)^{-1 / 2} \exp \left(i \int_{t_{0}}^{t} \Omega_{k}^{(n)}\left(t^{\prime}\right) d t^{\prime}\right)
$$

and call an adiabatic vacuum state a Fock state obtained from the solution of (2.40) with initial conditions at time $t$ given by

$$
T_{k}(t)=W_{k}^{(n)}(t), \quad \dot{T}_{k}(t)=\dot{W}_{k}^{(n)}(t) .
$$

The adiabatic vacuum states therefore depends on

a) the initial time $t$ in (3.6),

b) the order of iteration $n$,

c) the extrapolation of $\Omega^{(n)}$ to small values of $k$.

Changing the limit of integration $t_{0}$ in (3.5) merely changes the initial conditions (3.6) by a common phase and leaves the adiabatic vacuum state unchanged.

To be able to give conditions under which two adiabatic vacuum states lead to locally quasiequivalent representations, we need information on the asymptotic behaviour of $\Omega^{(n)}$ and $\varepsilon_{n}$ as $k$ goes to infinity, where

$$
\Omega^{(n)^{2}}=\Omega^{(n-1)^{2}}\left(1+\varepsilon_{n}\right) .
$$

A little manipulation leads to the following formulae

$$
\begin{aligned}
\frac{\dot{\Omega}^{(n)}}{\Omega^{(n)}}= & \frac{\dot{\Omega}^{(0)}}{\Omega^{(0)}}+\frac{1}{2} \frac{\dot{\varepsilon}_{1}}{1+\varepsilon_{1}}+\cdots+\frac{1}{2} \frac{\dot{\varepsilon}_{n}}{1+\varepsilon_{n}}, \\
\varepsilon_{n+1}= & \frac{1}{\Omega^{(0)^{2}}} \frac{1}{\left(1+\varepsilon_{1}\right) \cdots\left(1+\varepsilon_{n}\right)}\left(\frac{1}{4} \frac{\dot{\Omega}^{(0)}}{\Omega^{(0)}} \frac{\dot{\varepsilon}_{n}}{1+\varepsilon_{n}}+\frac{1}{8} \frac{\dot{\varepsilon}_{1}}{1+\varepsilon_{1}} \frac{\dot{\varepsilon}_{n}}{1+\varepsilon_{n}}+\cdots\right. \\
& \left.+\frac{1}{8} \frac{\dot{\varepsilon}_{n-1}}{1+\varepsilon_{n-1}} \frac{\dot{\varepsilon}_{n}}{1+\varepsilon_{n}}+\frac{5}{16} \frac{\dot{\varepsilon}_{n}^{2}}{1+\varepsilon_{n}}-\frac{1}{4} \frac{\ddot{\varepsilon}_{n}}{1+\varepsilon_{n}}\right)
\end{aligned}
$$

To describe the asymptotic behaviour we work over a fixed but arbitrary closed 
interval of time $I=\left[t_{0}, t_{1}\right]$ and, given a function $f(k, t)$ defined for $k \geqq k_{f}$ and $t \in I$, we write $f \in \mathscr{Q}_{n}(I)$ to mean that there exist constants $c_{m}>0, m=0,1,2, \ldots$ and $b>0$ such that

$$
\left|f^{(m)}(k, t)\right| \leqq c_{m} k^{n}, t \in I, k \geqq b .
$$

In other words, $f \in \mathscr{Q}_{n}(I)$ if $f$ and each of its derivatives with respect to $t$ are $O\left(k^{n}\right)$ for $k \rightarrow \infty$ uniformly in $t \in I$.

Lemma 3.2. We have $\Omega^{(n)} \in \mathscr{Q}_{1}(I)$ and $\varepsilon_{n} \in \mathscr{Q}_{-2 n}(I)$.

Proof. To simplify notation, we suppress the dependence on the interval $I$. Note that $f \in \mathscr{Q}_{n}$ and $f^{\prime} \in \mathscr{Q}_{n^{\prime}}$ implies $f f^{\prime} \in \mathscr{Q}_{n+n^{\prime}}$. Now $\Omega^{(0)^{2}} \in \mathscr{Q}_{2}$ and $\Omega^{(0)^{-2}} \in \mathscr{Q}_{-2}$ giving

$$
\frac{\dot{\Omega}^{(0)}}{\Omega^{(0)}} \in \mathscr{Q}_{0} \text { and } \frac{\ddot{\Omega}^{(0)}}{\Omega^{(0)}} \in \mathscr{Q}_{0} \text {. }
$$

It now follows taking $n=0$ in (3.3) that $\Omega^{(0)^{2}} \varepsilon_{1} \in \mathscr{Q}_{0}$ and hence $\varepsilon_{1} \in \mathscr{Q}_{\mathrm{s}_{2}}$. We now claim inductively that $\varepsilon_{n} \in \mathscr{Q}_{-2 n}$. This implies $\left(1+\varepsilon_{n}\right)^{-1} \in \mathscr{Q}_{0}$ and from (3.9) we get $\varepsilon_{n+1} \in \mathscr{Q}_{-2 n-2}$ completing the induction step. It follows at once that $\Omega^{(n)} \in \mathscr{Q}_{1}$ as required.

The assertions of Lemma 3.1 follow simply from Lemma 3.2 and (3.7). Now any two adiabatic vacuum states $\omega$ and $\omega^{\prime}$ differ by a Bogoliubov transformation and if we parametrize these states using functions $q(k), p(k)$ and $q^{\prime}(k), p^{\prime}(k)$ respectively, (cf. (2.48)), then we have the relations

$$
q^{\prime}(k)=\alpha(k) q(k)+\beta(k) \overline{q(k)}, \quad p^{\prime}(k)=\alpha(k) p(k)+\beta(k) \overline{p(k)},
$$

where the Bogoliubov coefficients $\alpha$ and $\beta$ satisfy

$$
|\alpha(k)|^{2}-|\beta(k)|^{2}=1 \text {. }
$$

Using (2.36), we may solve for $\beta$ and get

$$
\beta(k)=i\left(p^{\prime}(k) q(k)-q^{\prime}(k) p(k)\right) .
$$

It follows from Lemma 3.2 that for all adiabatic vacuum states $q(k)=O\left(k^{-1 / 2}\right)$ and $p(k)=O\left(k^{1 / 2}\right)$ as $k \rightarrow \infty$. For this reason, we obtain sufficient conditions for global unitary equivalence or local quasiequivalence that can be expressed solely in terms of the behaviour of $\beta(k)$ as $k \rightarrow \infty$. In the case of a closed Robertson-Walker spacetime, $\varepsilon=+$, where $k$ takes on discrete values, we show in Sect. 4 that we have global unitary equivalence if $\beta(k)=O\left(k^{-(3 / 2)-\delta}\right)$ for some $\delta>0$. In the case of an open Robertson-Walker spacetime, $\varepsilon=0$, -, where $k$ is continuous we show that we have local quasiequivalence if $\beta(k)=O\left(k^{-3-\delta}\right)$ for some $\delta>0$. The power $k^{-3}$ instead of the $k^{-3 / 2}$ one might expect may well prove to be an artefact of our method of proof.

We now consider the various factors affecting the adiabatic vacuum states. Extrapolating $\Omega^{(n)}$ differently to small values of $k$ corresponds to a Bogoliubov transformation, where $\beta(k)$ vanishes for sufficiently large $k$ and poses no problems. To assess the effects of the order of iteration we write $\Omega:=\Omega^{(n)}$ and $\Omega^{\prime}:=\Omega^{(n+1)}$ and compute $\beta$ from (3.12) and find

$$
\beta=\frac{1}{4}\left(\Omega \Omega^{\prime}\right)^{-(1 / 2)}\left[2\left(\Omega-\Omega^{\prime}\right)-i\left(\frac{\dot{\Omega}^{\prime}}{\Omega^{\prime}}-\frac{\dot{\Omega}}{\Omega}\right)\right] e^{i s \Omega} e^{i \int \Omega^{\prime}},
$$


where, to be concise, we have omitted the variables. Introducing $\varepsilon_{n+1}$ and using Lemma 3.2 we see that $\beta(k)=O\left(k^{-2 n-2}\right)$. Thus for closed Robertson-Walker spacetime the global unitary equivalence class is independent of the order of iteration, for open Robertson-Walker spacetimes the local quasiequivalence class becomes independent of the order of iteration after a single iteration. This leaves open the question of the dependence on the intitial time $t$ in (3.6).

Suppose we have a solution $T$ of our differential equation (2.40) with initial conditions

$$
T\left(t_{1}\right)=W\left(t_{1}\right), \quad \dot{T}\left(t_{1}\right)=\dot{W}\left(t_{1}\right),
$$

where we have suppressed the dependence on $k$ and, cf. (3.5),

$$
W(t)=a^{-3 / 2}(t)(2 \Omega(t))^{-1 / 2} \exp \left(i \int_{t_{0}}^{t} \Omega\left(t^{\prime}\right) d t^{\prime}\right) .
$$

Then at time $t$ this solution has initial conditions $T(t)$ and $\dot{T}(t)$ differing, in general, from $W(t)$ and $\dot{W}(t)$ and derived from $W(t)$ and $\dot{W}(t)$ by a Bogoliubov transformation with coefficients given by

$$
\alpha(t)=i a^{3}(t)(T(t) \dot{\bar{W}}(t)-\dot{T}(t) \bar{W}(t)), \quad \beta(t)=i a^{3}(t)(\dot{T}(t) W(t)-T(t) \dot{W}(t)) .
$$

Now, as was shown by Parker [13; Eq. (26)], $\alpha$ and $\beta$ satisfy the following coupled integral equations:

$$
\begin{aligned}
& \alpha(t)=1-i \int_{t_{1}}^{t} S\left(t^{\prime}\right)\left[\alpha\left(t^{\prime}\right)+\beta\left(t^{\prime}\right) \exp \left(-2 i \int_{t_{0}}^{t^{\prime}} \Omega\left(t^{\prime \prime}\right) d t^{\prime \prime}\right)\right] d t^{\prime}, \\
& \beta(t)=i \int_{t_{1}}^{t} S\left(t^{\prime}\right)\left[\beta\left(t^{\prime}\right)+\alpha\left(t^{\prime}\right) \exp \left(2 i \int_{t_{0}}^{t^{\prime}} \Omega\left(t^{\prime \prime}\right) d t^{\prime \prime}\right)\right] d t^{\prime},
\end{aligned}
$$

where the kernel $S$ is defined by

$$
2 S \Omega=\Omega^{2}-\frac{3}{4}\left(\frac{\dot{\Omega}}{\Omega}\right)^{2}+\frac{1}{2} \frac{\ddot{\Omega}}{\Omega}-\omega^{2}+\frac{3}{4}\left(\frac{\dot{a}}{a}\right)^{2}+\frac{3}{2} \frac{\ddot{a}}{a} .
$$

In particular, if we take $\Omega=\Omega^{(n)}$ then we find from (3.3) and (3.7) that the corresponding kernel $S^{(n)}$ is given by

$$
S^{(n)}=-\frac{1}{2} \Omega^{(n)} \varepsilon_{n+1} .
$$

It now follows from Lemma 3.2 that $S^{(n)} \in \mathscr{Q}_{-2 n-1}(I)$.

Standard arguments from the theory of integral equations of Volterra type sketched in an appendix then show that $\beta(k)=O\left(k^{-2 n-1}\right)$ uniformly for $t \in I$ and that $\alpha$ and $\beta$ are continuous as functions of $k$ uniformly in $t \in I$. From this result we may, for example, conclude directly that, in a Robertson-Walker spacetime with flat spatial sections, the initial time $t$ in (3.6) does not affect the local quasiequivalence class for an iteration order $\geqq 2$. Since, however, the local quasiequivalence class is anyway already known to be independent of the iteration order after a single iteration, we conclude indirectly that the local quasiequivalence class is independent of the initial time for an iteration order $\geqq 1$. Similar remarks hold in the other two cases. Hence our discussion may be summed up in the following result. 
Theorem 3.3. The following statements hold for the free Klein-Gordon field.

a) In a Robertson-Walker spacetime with spherical spatial sections any two adiabatic vacuum states are unitarily equivalent.

b) In an open Robertson-Walker spacetime any two adiabatic vacuum states of iteration order $\geqq 1$ are locally quasiequivalent.

A result in this direction is foreshadowed in Footnote 26 of a paper by Parker and Fulling [14]. There are no obvious obstacles to extending this analysis to cover the case of a Klein-Gordon field coupled to the scalar curvature, i.e. to an equation of the form

$$
\left(\square+m^{2}+\xi R\right) \varphi=0
$$

\section{Quasiequivalence of Quasifree States}

In this section we derive conditions on the Bogoliubov coefficients $\beta$ of (3.12) which, together with the factoriality of $\pi_{\omega} \uparrow \mathcal{O}$ (for certain $\mathcal{O}$ ), proved in Sect. 5, guarantee that two homogeneous, isotropic Fock states $\omega$ and $\omega^{\prime}$, characterized by functions $p, q$ and $p^{\prime}, q^{\prime}$ respectively, are locally quasiequivalent. If $\pi_{\omega} \uparrow \mathcal{O}$ and $\pi_{\omega^{\prime}} \uparrow \mathcal{O}$ are quasiequivalent then so are their restrictions to some $\mathcal{O}_{1} \subset \mathcal{O}$. It therefore suffices to verify the local quasiequivalence for a cofinal set of bounded open sets $\mathcal{O}$. For this reason we can choose $\mathcal{O}$ to have simple geometric properties which make it easier to check the criterion of Araki and Yamagami (cf. Theorem 1.1) and to demonstrate the factoriality of $\pi_{\omega} \uparrow \mathcal{O}$.

In the case of a closed universe $(\varepsilon=+)$, where the Cauchy surfaces $\mathscr{S}_{t}$ are compact, we can even choose $\mathcal{O}=\mathscr{M}$ and then give a necessary and sufficient condition on $\beta$ for the global unitary equivalence of the Fock states. For this reason this case is much simpler than the case of an open universe where global unitary equivalence is only possible when $\beta=0$. We therefore begin by treating a closed universe and we want to apply Theorem 1.1 to the Weyl algebra corresponding to the phase space $K=\mathscr{D}(\mathscr{M}) /\left(\square+m^{2}\right) \mathscr{D}(\mathscr{M})$. It is equivalent and technically more convenient to work, as in Sect. 2, with the isomorphic phase space of initial data $L$.

We begin with Condition (A1) of Theorem 1.1 and, with an eye to applications in Sect. 5, give conditions for a scalar product $(\cdot, \cdot)_{S}$ defined by (1.9), (2.22), (2.23) and $(2.35)$ to be equivalent to the scalar product $(\cdot,)_{M}$ given by

$$
(F, G)_{M}=\int d \vec{k}\langle\tilde{F}(\vec{k}), M(k) \tilde{G}(\vec{k})\rangle \text {, where } \quad M(k):=2\left(\begin{array}{cc}
|q(k)|^{2} & 0 \\
0 & |p(k)|^{2}
\end{array}\right) .
$$

Lemma 4.1. Given functions $q$ and $p$ satisfying $(2.36)$ with $q(k)=O\left(k^{-1 / 2}\right)$ and $p(k)=O\left(k^{1 / 2}\right)$, the scalar products $(\cdot,)_{S}$ and $(\cdot,)_{M}$ define the same topology on $L$, i.e. there are positive constants $A, B$ with

$$
A(F, F)_{M} \leqq(F, F)_{S} \leqq B(F, F)_{M} .
$$

Proof. The second inequality is satisfied if $B M(k)-\left(S(k)+S^{t}(k)\right)$ is a positive matrix and using (2.36) this is easily seen to be the case when $B \geqq 2$. Similarly, the first 
inequality is satisfied when

$$
0<A<1-\sqrt{1-1 / c}, \quad \text { where } \quad c=\max \left\{1, \sup _{k \in \mathbf{N}_{0}} 4|q(k)|^{2}|p(k)|^{2}\right\} .
$$

Remark. Lemma 4.1 is also valid when $\varepsilon=0$, - provided one requires $q$ and $p$ to be, for example, continuous in $k$.

It is now clear that if we have a second Fock state $\omega_{S^{\prime}}$ corresponding to functions $q^{\prime}$ and $p^{\prime}$ given by (3.10), where the Bogoliubov coefficient $\beta$ is bounded, then $(\cdot, \cdot)_{S}$ and $(\cdot, \cdot)_{S^{\prime}}$ define the same topology.

We now turn to Condition (A2) and let $U: L_{S} \rightarrow L^{2}(\tilde{\mathscr{S}}) \oplus L^{2}(\tilde{\mathscr{S}})$ be the unitary operator such that

$$
U F=N\left(\begin{array}{c}
\# f \\
\tilde{h}
\end{array}\right), \quad N(k):=\left(\frac{q(k)}{q(k)} \frac{p(k)}{p(k)}\right), \quad F=\left(\begin{array}{l}
f \\
h
\end{array}\right) \in L .
$$

A computation shows that

$$
U \tilde{S} U^{*}=N^{-1 *} S N^{-1}=\left(\begin{array}{ll}
1 & 0 \\
0 & 0
\end{array}\right),
$$

where $S$ here denotes multiplication with the matrix $S(k)$ of $(2.22)$. We would get the same result if we were to compute $N^{\prime-1 *} S^{\prime} N^{\prime-1}$, where

$$
N^{\prime}=\left(\begin{array}{cc}
q^{\prime} & p^{\prime} \\
\bar{q}^{\prime} & \bar{p}^{\prime}
\end{array}\right)=\left(\begin{array}{cc}
\alpha & \beta \\
\bar{\beta} & \bar{\alpha}
\end{array}\right)\left(\begin{array}{ll}
q & p \\
\bar{q} & \bar{p}
\end{array}\right) \text {. }
$$

Thus

$$
U{\tilde{S^{\prime}}}^{*} U^{-1 *} S^{\prime} N^{-1}=\left(\begin{array}{cc}
|\alpha|^{2} & \bar{\alpha} \beta \\
\bar{\beta} \alpha & |\beta|^{2}
\end{array}\right) .
$$

(A2) is then equivalent to $\Delta:=\left(U \tilde{S}^{\prime} U^{*}\right)^{1 / 2}-\left(U \tilde{S} U^{*}\right)^{1 / 2}$ being Hilbert-Schmidt. The square roots can be easily computed since the second term is a projection whilst the first term is just a multiple of a projection. Using $|\alpha|^{2}-|\beta|^{2}=1$, we obtain

$$
\Delta=|\beta| A \text {, where } A=\left(1+2|\beta|^{2}\right)^{-1 / 2}\left(\begin{array}{cc}
{\left[1-\left(1+2|\beta|^{2}\right)^{1 / 2}\right]|\beta|^{-1}+|\beta|} & \bar{\alpha} \beta /|\beta| \\
\alpha \bar{\beta} /|\beta| & |\beta|
\end{array}\right) .
$$

Provided $\beta$ is bounded, $A$ is a bounded operator with bounded inverse and $\Delta$ is Hilbert-Schmidt if and only if

$$
\operatorname{Tr}|\beta|^{2}=2 \sum_{k=0}^{\infty} \sum_{l=0}^{k} \sum_{m=-l}^{l}|\beta(k)|^{2}=2 \sum_{k=0}^{\infty}(k+1)^{2}|\beta(k)|^{2}<+\infty .
$$

Thus for the closed universe we get global unitary equivalence, and hence also local quasiequivalence if

$$
|\beta(k)| \leqq C(1+k)^{-(3 / 2)-\delta}, \quad \delta>0 .
$$

The case of an open universe, $\varepsilon=0$, - is more complicated. The multiplication operator $\beta$ has a continuous spectrum for $\beta \neq 0$ and is not a Hilbert-Schmidt operator. Hence the corresponding Fock states are not unitarily equivalent. We shall therefore need to apply Theorem 1.1 to the Weyl algebra over the phase space 
$(K(\mathcal{O}), \Gamma, \gamma)$, where $K(\mathcal{O})$ denotes the image of $\mathscr{D}(\mathcal{O})$ in $K=\mathscr{D}(\mathscr{M}) /\left(\square+m^{2}\right) \mathscr{D}(\mathscr{M})$. To be able to identify $K(\mathcal{O})$ in a simple manner with a subspace of $L_{S}$ we choose $\mathcal{O}$ to be the causal closure of a subset $\mathscr{C}$ of a Cauchy surface $\mathscr{S}_{t}$. Thus $\mathcal{O}=D(\mathscr{C})=D^{+}(\mathscr{C}) \cup D^{-}(\mathscr{C})$, where $D^{+}(\mathscr{C})$ and $D^{-}(\mathscr{C})$ are the set of points $x \in \mathscr{M}$ such that each past or each future inextendible causal curve respectively intersects $\mathscr{C}$. We shall further assume that $\mathscr{C}$ is an open bounded subset of $\mathscr{S}_{t}$ with smooth boundary since we shall need these properties in Sect. 5 .

We next need to establish the relationship between $K(\mathcal{O}), \mathcal{O}=D(\mathscr{C})$, and $L(\mathscr{C}):=\{(f, h) \in L$ :supp $f \subset \mathscr{C}$, supp $h \subset \mathscr{C}\}$ and we show that the closure $L_{S}(\mathscr{C})$ of $L(\mathscr{C})$ in $L_{S}$ is just the image of the closure $K_{S}(\mathcal{O})$ of $K(\mathcal{O})$ in $K_{S}$ under the extension of $\rho_{t}$ to $K_{S}$, a unitary operator which we continue to denote by $\rho_{t}$.

Lemma 4.2. Let $\mathcal{O}=D(\mathscr{C})$, then $\rho_{t} K_{S}(\mathcal{O})=L_{S}(\mathscr{C})$.

Proof. The propagation properties of solutions of the Klein-Gordon equation [3] show that $\rho_{t} K(\mathcal{O}) \subset L(\mathscr{C})$. Hence $\rho_{t} K_{S}(\mathcal{O}) \subset L_{S}(\mathscr{C})$ and it suffices to show that $\rho_{t} K(\mathcal{O})$ is dense in $L_{S}(\mathscr{C})$. To this end, given $F=(f, h) \in L(\mathscr{C})$, we consider a sequence $f_{n}$ of elements of $\mathscr{D}(\mathscr{M})$, where

$$
\left(\# f_{n}\right)(x)=-a^{-3}\left(x^{0}\right)\left\{d_{n}\left(x^{0}-t\right)(\# f)(\vec{x})-\dot{d}_{n}\left(x^{0}-t\right) h(\vec{x}) a^{3}(t)\right\}
$$

and $d \in C_{0}^{\infty}(\mathbb{R}), \int d(t) d t=1, d_{n}(t)=n d(n t)$. By writing $E$ in the form given in (2.44) we show that $\rho_{t} f_{n} \rightarrow F$ as $n \rightarrow \infty$ in $L_{S}(\mathscr{C})$. With the help of the Fourier inversion formula (A.8) we get

$$
\begin{aligned}
-\left(j_{t}^{*} \widetilde{E f_{n}}\right)(\vec{k})= & \int d y^{0} G_{k}\left(t, y^{0}\right)\left\{d_{n}\left(y^{0}-t\right)(\widetilde{\# f})(\vec{k})-\dot{d}_{n}\left(y^{0}-t\right) a^{3}(t) \tilde{h}(\vec{k})\right\} \\
= & \int d y^{0}\left\{G_{k}\left(t, t+\frac{y^{0}}{n}\right)(\# f)(\vec{k})+a^{3}(t)\left(\partial_{2} G_{k}\right)\left(t, t+\frac{y^{0}}{n}\right) \tilde{h}(\vec{k})\right\} d\left(y^{0}\right), \\
\left(\# j_{t}^{*} \# \tilde{d E} f_{n}\right)(\vec{k})= & -\int d y^{0}\left\{a^{3}(t) \partial_{1} G_{k}\left(t, t+\frac{y^{0}}{n}\right)(\widetilde{\# f})(\vec{k})\right. \\
& \left.+a^{6}(t)\left(\partial_{1} \partial_{2} G_{k}\right)\left(t, t+\frac{y^{0}}{n}\right) \tilde{h}(\vec{k})\right\} d\left(y^{0}\right),
\end{aligned}
$$

where $\partial_{j}$ denotes differentiation with respect to the $j^{\text {th }}$ variable. Since $G_{k}\left(x^{0}, y^{0}\right)$ is polynomially bounded in $k$ uniformly for $x^{0}, y^{0}$ in a compact interval (cf. Appendix C) and hence in particular bounded in $y^{0}$ on the support of $d$ for fixed $x^{0}$ and $k$, the dominated converges theorem together with (2.43) and (2.41) show that $\rho_{t} f_{n}$ tends to $(f, h)$ in $L_{S}(\mathscr{C})$ as required. Furthermore, $f_{n}$ has support in $\mathcal{O}$ for $n$ sufficiently large, completing the proof.

Lemma 4.2 could also be deduced from Lemma A.3 of [3]. We are now in a position to investigate local quasiequivalence using the phase space of initial data. As in Lemma 4.1, one may can show that continuous functions $q, p$ and $q^{\prime}, p^{\prime}$ with $p(k)=O\left(k^{1 / 2}\right), q(k)=O\left(k^{-1 / 2}\right), p^{\prime}(k)=O\left(k^{1 / 2}\right), q^{\prime}(k)=O\left(k^{-1 / 2}\right)$ yield polarizations $S$ and $S^{\prime}$ satisfy (A1). To demonstrate the quasiequivalence of the restrictions of $\omega_{S}$ and $\omega_{S^{\prime}}$ to the Weyl algebra over $L(\mathscr{C})$ we must consider the operators $E \widetilde{S} E$, and $E \tilde{S^{\prime}} E$, where $E$ denotes the projection in $L_{S}$ onto the closed subspace $L_{S}(\mathscr{C})$ and verify that

$$
(E \tilde{S} E)^{1 / 2}-\left(E \tilde{S}^{\prime} E\right)^{1 / 2} \in \mathscr{L}^{2}\left(L_{S}\right)
$$


Since the square roots cannot now be computed explicitly, we fall back on a sufficient condition for (4.6) to hold given in [15]. To this end, we pick $\chi \in C_{0}^{\infty}(\mathscr{S})$ with $\chi \geqq 0$ and $\chi\lceil\mathscr{C}=1$. As we shall show in Sect. 5, there is a bounded operator $\chi$ on $L_{S}$ such that $\chi F=(\chi f, \chi h)$ and obviously $\chi E=E$. We now have

Lemma 4.3 [15]. If $\chi^{*}\left(\tilde{S}-\tilde{S}^{\prime}\right) \chi \in \mathscr{L}^{1}\left(L_{S}\right)$ then (4.6) holds.

Here $\mathscr{L}^{1}\left(L_{S}\right)$ denotes the set of trace class operators on $L_{S}$.

We shall actually show that $T:=U \chi^{*}\left(\tilde{S}-\widetilde{S}^{\prime}\right) \chi U^{*}$ is trace class, where $U$ is the unitary operator $U: L_{S} \rightarrow L^{2}(\widetilde{\mathscr{S}}) \oplus L^{2}(\tilde{\mathscr{S}})$ defined by (4.2). Now using $|\alpha|^{2}-|\beta|^{2}=1$ we get

$$
U\left(\tilde{S^{\prime}}-\tilde{S}\right) U^{*}=\left(\begin{array}{cc}
|\beta|^{2} & \bar{\alpha} \beta \\
\bar{\beta} \alpha & |\beta|^{2}
\end{array}\right),
$$

(cf. (4.3) and (4.4)). $U \chi U^{*}$ is given by an integral kernel of the form

$$
N(k)\left(\begin{array}{cc}
\hat{\chi}\left(\vec{k}, \vec{k}^{\prime}\right) & 0 \\
0 & \hat{\chi}\left(\vec{k}, \vec{k}^{\prime}\right)
\end{array}\right) N\left(k^{\prime}\right)^{-1}
$$

where

$$
\hat{\chi}\left(\vec{k}, \vec{k}^{\prime}\right)=\int d \mu(\vec{x}) \overline{\mathscr{Y}}_{\vec{k}}(\vec{x}) \chi(\vec{x}) \mathscr{Y}_{\vec{k}^{\prime}}(\vec{x}),
$$

and $\mu$ is the invariant measure derived from the Riemannian metric. We show that the integral operator $T$ has trace class by writing it as the product of two Hilbert-Schmidt operators $T=T_{1}^{*} T_{2}$,

$$
T_{1}=\left(\begin{array}{cc}
|\beta|^{1 / 2} & 0 \\
0 & |\beta|^{1 / 2}
\end{array}\right) U \chi U^{*}, \quad T_{2}=\left(\begin{array}{cc}
1 & \bar{\alpha} \beta /|\beta| \\
\alpha \bar{\beta} /|\beta| & 1
\end{array}\right) T_{1} .
$$

Since $\left(\begin{array}{cc}1 & \bar{\alpha} \beta /|\beta| \\ \alpha \bar{\beta} /|\beta| & 1\end{array}\right)$ is a bounded operator for $\beta$ bounded and continuous, we have only to show that $T_{1}$ is Hilbert-Schmidt. This is the case when the integral operators given by

$$
I_{+}\left(\vec{k}, \vec{k}^{\prime}\right)=|\beta(k)|^{1 / 2} q(k) \hat{\chi}\left(\vec{k}, \vec{k}^{\prime}\right) p\left(k^{\prime}\right), \quad I_{-}\left(\vec{k}, \vec{k}^{\prime}\right)=|\beta(k)|^{1 / 2} p(k) \hat{\chi}\left(\vec{k}, \vec{k}^{\prime}\right) q\left(k^{\prime}\right),
$$

are Hilbert-Schmidt, i.e. when $I_{ \pm} \in L^{2}(\tilde{\mathscr{S}} \times \tilde{\mathscr{S}})$. In order to estimate $I_{ \pm}$, we use

$$
|q(k)|^{2} \leqq c_{-}(1+k)^{-1}, \quad|p(k)|^{2} \leqq c_{+}(1+k) .
$$

In the case $\varepsilon=0$, we have

$$
\begin{aligned}
\hat{\chi}\left(\vec{k}, \vec{k}^{\prime}\right) & =(2 \pi)^{-3 / 2} \tilde{\chi}\left(\vec{k}-\vec{k}^{\prime}\right), \quad \tilde{\chi} \in \mathscr{S}\left(\mathbb{R}^{3}\right), \\
\left(1+\left|\vec{k}-\vec{k}^{\prime}\right|\right)^{ \pm 1} & \leqq\left(1+\left|\vec{k}^{\prime}\right|\right)(1+|\vec{k}|)^{ \pm 1} .
\end{aligned}
$$

We may therefore estimate

$$
\begin{aligned}
& \int d \vec{k} d \vec{k}^{\prime}\left|I_{ \pm}\left(\vec{k}, \vec{k}^{\prime}\right)\right|^{2} \leqq c_{+} c_{-}(2 \pi)^{-3} \int d \vec{k} d \vec{k}^{\prime}|\beta(k)|(1+|\vec{k}|)^{\mp 1}\left|\tilde{\chi}\left(\vec{k}^{\prime}\right)\right|^{2}\left(1+\left|\vec{k}-\vec{k}^{\prime}\right|\right)^{ \pm 1} \\
& \leqq c_{+} c_{-}(2 \pi)^{-3} \int d \vec{k} d \vec{k}^{\prime}|\beta(k)|\left|\tilde{\chi}\left(\vec{k}^{\prime}\right)\right|^{2}\left(1+\left|\vec{k}^{\prime}\right|\right) .
\end{aligned}
$$

It now follows that $I_{ \pm}$is Hilbert-Schmidt if $k \mapsto \beta(k)$ is continuous and

$$
|\beta(k)| \leqq c(1+k)^{-3-\delta}, \quad \delta>0 .
$$


It could well be that the extra powers of $k$ as compared with (4.5) are a result of using Lemma 4.3.

In the case $\varepsilon=-$, we do not have the simple formula (4.13) at our disposal and we need to apply some estimates on the Fourier transform obtained using the method of stationary phase. Thus we use Theorem 7.7.1 of [16] to prove that, for each $N \in \mathbb{N}_{0}$, there is a $c_{N}>0$ such that

$$
\left|\hat{\chi}\left(\vec{k}, \vec{k}^{\prime}\right)\right| \leqq c_{N}\left(1+\left|\vec{k}-\vec{k}^{\prime}\right|\right)^{-N} .
$$

The proof is relegated to Appendix D. The argument in the case $\varepsilon=0$ is now easily adapted to show that $I_{ \pm}$are Hilbert-Schmidt whenever $|\beta(k)| \leqq c(1+k)^{-3-\delta}, \delta>0$.

We may therefore summarize the results of this section as follows:

Theorem 4.5. Given two homogeneous isotropic Fock states $\omega$ and $\omega^{\prime}$ characterized by functions $p, q$ and $p^{\prime}, q^{\prime}$ respectively, where $q(k)=O\left(k^{-1 / 2}\right)$ and $p(k)=O\left(k^{1 / 2}\right)$, let $\beta(k)$ be defined by (3.12) then

a) In a closed Robertson-Walker spacetime $(\varepsilon=+) \pi_{\omega}$ and $\pi_{\omega^{\prime}}$ are unitarily equivalent if $|\beta(k)| \leqq c(1+k)^{-(3 / 2)-\delta}, \delta>0$.

b) In an open Robertson-Walker spacetime $(\varepsilon=0,-)$ the representations $\pi_{\omega \backslash \mathbb{C}}$ and $\pi_{\omega^{\prime} \uparrow \mathcal{O}}$ are quasiequivalent for $\mathcal{O}=D(\mathscr{C})$ provided $p, q$ and $p^{\prime}, q^{\prime}$ are continuous and $|\beta(k)| \leqq c(1+k)^{-3-\delta}, \delta>0$.

This result, together with the factoriality of $\pi_{\omega} \uparrow \mathcal{O}$, to be proved in the next section for adiabatic vacuum states, leads to Theorem 3.3.

\section{Factoriality of the Local Algebras}

In this section we complete the proof of local quasiequivalence for the adiabatic vacuum states by showing that $\pi_{\omega} \uparrow \mathcal{O}$ is factorial when $\mathcal{O}=D(\mathscr{C})$ and $\mathscr{C}$ is an open bounded subset of $\mathscr{S}_{t}$ with smooth boundary. Of course, we only need this result for open Robertson-Walker spacetimes and our method of proof is to reduce to the case of the Fock vacuum state in Minkowski space, where the result is well known [17].

As in the last section, we work with the phase space of initial data $(L, \Gamma, \gamma)$ and we let $\mathfrak{R}_{S}(\mathscr{C})$ denote the von Neumann algebra generated by the Weyl operators $W(F), F=\Gamma F \in L_{S}(\mathscr{C})$, in the irreducible representation corresponding to the Fock state $\omega_{S}$. We must show that $\mathfrak{R}_{S}(\mathscr{C})$ is a factor, i.e. that $\mathfrak{R}_{S}(\mathscr{C}) \cap \mathfrak{R}_{S}(\mathscr{C})^{\prime}=\mathbb{C} I$, and we use the following well known result, cf. [4; Theorem 3.12],

Theorem 5.1. $\mathfrak{R}_{S}(\mathscr{C})$ is a factor if and only if

$$
L_{S}(\mathscr{C}) \cap L_{S}(\mathscr{C})^{\prime}=0,
$$

where $L_{S}(\mathscr{C})^{\prime}$ denotes the symplectic complement of $L_{S}(\mathscr{C})$,

$$
L_{S}(\mathscr{C})^{\prime}:=\left\{F \in L_{S}: \gamma\left(F, F^{\prime}\right)=0, F^{\prime} \in L_{S}(\mathscr{C})\right\},
$$

and we have extended $\gamma$ by continuity to $L_{S}$.

Now $L_{S}$ is, by Lemma 4.1, naturally isomorphic as a topological vector space to the Hilbert space $H:=H_{-\sigma} \oplus H_{\sigma}, \sigma=\frac{1}{2}$, where $H_{\sigma}$ denotes the completion of 
$C_{0}^{\infty}(\mathscr{S})$ with respect to the scalar product

$$
(f, g)_{\sigma}=\int d \vec{k} \omega_{k}^{2 \sigma} \overline{\tilde{f}}(\vec{k}) \tilde{g}(\vec{k}), \quad f, g \in C_{0}^{\infty}(\mathscr{S}) .
$$

Furthermore, the closed subspace of $H$ corresponding to $L_{S}(\mathscr{C})$ will be denoted $H(\mathscr{C}):=H_{-\sigma}(\mathscr{C}) \oplus H_{\sigma}(\mathscr{C})$. The operators $\omega^{\sigma}$ acting as multiplication operators in momentum space will be considered as unitary operators from $H_{\sigma}$ to $L^{2}(\mathscr{S})$. The Hermitian form $\gamma$ of (2.7) can now be written

$$
\gamma\left(F, F^{\prime}\right)=i\left(\omega^{-1 / 2} \# f, \omega^{1 / 2} h^{\prime}\right)-i\left(\omega^{1 / 2} h, \omega^{-1 / 2} \# f^{\prime}\right), \quad F, F^{\prime} \in L .
$$

Hence (5.1) is equivalent to

$$
H_{\sigma}(\mathscr{C})^{\prime} \cap H_{-\sigma}(\mathscr{C})=0 \text { for } \sigma= \pm \frac{1}{2}
$$

where

$$
H_{\sigma}(\mathscr{C})^{\prime}=\left\{f \in H_{-\sigma}:\left(\omega^{-\sigma} f, \omega^{\sigma} g\right)=0, g \in H_{\sigma}(\mathscr{C})\right\} .
$$

In the case $\varepsilon=0$ the spaces $H_{\sigma}(\mathscr{C})$ and their symplectic complements take the same form as for the Minkowski vacuum $\omega_{0}$. Hence (5.5) for $\varepsilon=0$ is equivalent to the factoriality of $\pi_{\omega_{0}} \uparrow \mathcal{O}, \mathcal{O}=D(\mathscr{C})$. This property of the Minkowski vacuum representation was shown by Araki [17]. As Araki defines the local algebras in an a priori different manner and as his proof invokes duality, we present here a proof whose basic idea goes back to an unpublished seminar of J. Bellissard in 1976. The elements of $H_{\sigma}$ can be considered in the usual manner as distributions from $\mathscr{D}^{\prime}\left(\mathbb{R}^{3}\right)$ and $H_{\sigma}$ is continuously embedded in $\mathscr{D}^{\prime}\left(\mathbb{R}^{3}\right)$. Thus the set of elements of $H_{\sigma}$ with support in a closed set is a closed subspace of $H_{\sigma}$. It follows that the elements of $H_{\sigma}(\mathscr{C})$ have support in the closure of $\mathscr{C}$. On the other hand, it follows straight from the definition that the elements of $H_{\sigma}(\mathscr{C})^{\prime}$ have supports contained in the complement of $\mathscr{C}$. Hence the support of a distribution in $H_{\sigma}(\mathscr{C}) \cap H_{-\sigma}(\mathscr{C})^{\prime}$ is contained in $\partial \mathscr{C}$, the boundary of $\mathscr{C}$. By assumption $\mathscr{C}$ has a smooth boundary and it is therefore locally diffeomorphic to a open subset of a hyperplane, for example $x^{3}=0$. Now the multiplication with $C_{0}^{\infty}$-functions and the transformation by local diffeomorphisms are continuous operations in $H_{\sigma}$ [18; Theorems 2.2 .5 and 2.6.1]. Thus, using a partition of the identity and local diffeomorphisms, an $h \in H_{\sigma}(\mathscr{C}) \cap H_{-\sigma}(\mathscr{C})^{\prime}$ gives rise to a set $h_{i} \in H_{\sigma}, i=1,2, \ldots, k$, with support in the hyperplane $x^{3}=0$ such that $h=0$ if and only if $h_{i}=0$ for all $i$. Now a distribution $\varphi$ with support in $x^{3}=0$ has the form $\varphi(\vec{x})=\sum_{|\alpha| \leqq j} \varphi_{\alpha}\left(x^{1}, x^{2}\right) \partial^{\alpha} \delta\left(x^{3}\right)$ [16; Theorem 2.3.5, Ex. 5.1.2]. The Fourier transforms of the $h_{i}$ are constant or of polynomial growth in $k_{3}$ and can therefore only be elements of $H_{\sigma}$ for $\sigma= \pm \frac{1}{2}$ if they are zero.

Remark. Since $L_{S}$ and $H=H_{-1 / 2} \oplus H_{1 / 2}$ are naturally isomorphic as topological vector spaces and multiplication by a $C_{0}^{\infty}$-function is continuous in $H_{\sigma}$, the operator $\chi$ defined in connection with Lemma 4.3 is bounded in the case $\varepsilon=0$.

In the case $\varepsilon=-$, we again regard an element $h$ of $H_{\sigma}$ as the distribution $f \mapsto\left(\omega^{-\sigma} f, \omega^{\sigma} h\right), f \in C_{0}^{\infty}(\mathscr{S})$ and as in the case $\varepsilon=0$ it follows that if $h \in H_{\sigma}(\mathscr{C}) \cap$ $H_{-\sigma}(\mathscr{C})^{\prime}$ its support is in $\partial \mathscr{C}$. The proof of (5.5) will be reduced to the case $\varepsilon=0$ and to this end we regard $\mathscr{S}^{0}$ and $\mathscr{S}^{-}$as $\mathbb{R}^{3}$ using the coordinates $x^{i}, i=1,2,3$ and show that $H_{\sigma}^{-}(\mathscr{C}) \subset H_{\sigma}^{0}$ with a continuous inclusion mapping. As we have already shown that $H_{\sigma}^{0}$ has no non-zero elements with support in $\partial \mathscr{C},(5.5)$ for 
$\varepsilon=-$ will follow. To show that $H_{\sigma}^{-}(\mathscr{C}) \subset H_{\sigma}^{0}$ for $\sigma= \pm \frac{1}{2}$ we first compare $H_{\sigma}^{-}(\mathscr{C})$ and $H_{\sigma}^{0}(\mathscr{C})$ for $\sigma=0$ and $\sigma=1$ using direct estimates, then derive a result for $\sigma=\frac{1}{2}$ by interpolation and finally dualize to obtain a result for $\sigma=-\frac{1}{2}$.

Lemma 5.2. There are positive constants $A_{\sigma}, B_{\sigma}, \sigma=0,1$, such that

$$
A_{\sigma}\|h\|_{\sigma}^{0} \leqq\|h\|_{\sigma}^{-} \leqq B_{\sigma}\|h\|_{\sigma}^{0}, \quad h \in C_{0}^{\infty}(\mathscr{C}) .
$$

Proof. The determinant of the metric on $\mathscr{S}^{-}$is $g(\vec{x})=\left(1+\vec{x}^{2}\right)^{-1}$ in these coordinates and is strictly positive on each compact subset of $\mathbb{R}^{3}$. The inequalities for $\sigma=0$ are a direct consequence. We have

$$
(h, h)_{1}^{-}=\int d \vec{x} g^{1 / 2}\left\{a^{-2}(t) g^{i j} \partial_{i} \bar{h} \partial_{j} h+m^{2}|h|^{2}\right\}, \quad h \in C_{0}^{\infty}\left(\mathbb{R}^{3}\right),
$$

and it follows that $\|h\|_{1}^{-} \leqq B_{1}\|h\|_{1}^{0}, h \in C_{0}^{\infty}(\mathscr{C})$, since the coefficients of the metric $g^{i j}$ are continuous. Now the other estimate is a special case of the Garding inequality [19; Theorem 36.1]. The differential operator $P(\vec{x}, \vec{\partial})=\partial_{i} g^{1 / 2}(\vec{x}) g^{i j}(\vec{x}) \partial_{j}$ is uniformly strongly elliptic in $\mathscr{C}$, that is its principal part $P_{2}(\vec{x}, \vec{\partial}):=g^{1 / 2}(\vec{x}) g^{i j}(\vec{x}) \partial_{i} \partial_{j}$ satisfies for some $\alpha>0$,

thus

$$
\left|P_{2}(\vec{x}, \vec{\xi})\right| \geqq \alpha|\vec{\xi}|^{2}, \quad \vec{\xi} \in \mathbb{R}^{3}, \vec{x} \in \mathscr{C}
$$

$$
g^{1 / 2}(\vec{x}) g^{i j}(\vec{x}) \partial_{i} \bar{h}(\vec{x}) \partial_{j} h(\vec{x}) \geqq \alpha|\vec{\nabla} h|^{2}, \quad h \in C_{0}^{\infty}(\mathscr{C}) .
$$

This together with the first part of the proof shows that $A_{1}\|h\|_{1}^{0} \leqq\|h\|_{1}^{-}$as required.

To be able to prove the boundedness of the operator $\chi$ of Lemma 4.3 using interpolation theory, we need the following lemma.

Lemma 5.3. Let $\chi \in C_{0}^{\infty}\left(\mathbb{R}^{3}\right)$, then the mapping $h \mapsto \chi h, h \in C_{0}^{\infty}\left(\mathbb{R}^{3}\right)$, extends to a continuous operator $\chi_{\sigma}: H_{\sigma}^{-} \rightarrow H_{\sigma}^{-}$for $\sigma=0,1$.

Proof. The proof for $\sigma=0$ is trivial. Now

$$
g^{i j}\left(\partial_{i} \overline{\chi h}\right)\left(\partial_{j} \chi h\right)=g^{i j}\left\{\partial_{i} \bar{\chi} \partial_{j} \chi|h|^{2}+\partial_{i} \bar{h} \partial_{j} h|\chi|^{2}+\left(\overline{h \partial_{i} \chi}\right)\left(\chi \partial_{j} h\right)+\left(\overline{\chi \partial_{i} h}\right)\left(h \partial_{j} \chi\right)\right\},
$$

and using

we get

$$
\left|\bar{\xi}_{i} g^{i j} \eta_{j}\right| \leqq \frac{1}{2}\left\{\bar{\xi}_{i} g^{i j} \xi_{j}+\bar{\eta}_{i} g^{i j} \eta_{j}\right\}
$$

$$
(\chi h, \chi h)_{1}^{-}-m^{2}(\chi h, \chi h)_{0}^{-} \leqq 2 \int d \vec{x} g^{1 / 2} a^{-2}(t)\left(g^{i j} \partial_{i} \bar{h} \partial_{j} h|\chi|^{2}+g^{i j} \partial_{i} \bar{\chi} \partial_{j} \chi|h|^{2}\right) .
$$

Since $(\chi h, \chi h)_{0}^{-} \leqq c_{0}(h, h)_{0}^{-}$we get $(\chi h, \chi h)_{1}^{-} \leqq c_{1}(h, h)_{1}^{-}, h \in C_{0}^{\infty}\left(\mathbb{R}^{3}\right)$, as required.

As a corollary to these two lemmas we have

Corollary 5.4. Let $\chi \in C_{0}^{\infty}(\mathscr{C})$, then the mapping $h \mapsto \chi h$ extends to give continuous operators $\vec{\chi}_{\sigma}: H_{\sigma}^{-} \rightarrow H_{\sigma}^{0}$ and $\overleftarrow{\chi}_{\sigma}: H_{\sigma}^{0} \rightarrow H_{\sigma}^{-}, \sigma=0,1$.

The operator $\omega^{2}=m^{2}-a^{-2}(t) \Delta$ is essentially self-adjoint on $C_{0}^{\infty}\left(\mathscr{S}^{-}\right)$[9] and since $\omega^{2} \geqq m^{2} I, \omega^{2 \sigma}$ will also be essentially self-adjoint on $C_{0}^{\infty}\left(\mathscr{S}^{-}\right)$for $\sigma \leqq 1$. Thus the spaces $H_{\sigma}$ defined by completing $C_{0}^{\infty}(\mathscr{S})$ with respect to the scalar product (5.3) for $\sigma \in[0,1]$ are just the spaces defined by quadratic interpolation (cf. [20; Ch. VIII]) and we have

Corollary 5.5. The mappings $\chi_{\sigma}, \vec{\chi}_{\sigma}$ and $\bar{\chi}_{\sigma}$ extend uniquely from $H_{1}^{-}$or $H_{1}^{0}$ respectively to continuous operators for each $\sigma \in[-1,1]$. 
Proof. For $\sigma \in[0,1]$ the result follows by the functoriality of quadratic interpolation. If we identify the dual space of $H_{\sigma}^{-}$and $H_{\sigma}^{0}$ with $H_{-\sigma}^{-}$and $H_{-\sigma}^{0}$ respectively, then we have $\chi_{\sigma}^{\prime}=\chi_{-\sigma}, \vec{\psi}_{\sigma}^{\prime}=\overleftarrow{\chi}_{-\sigma}$ and $\overleftarrow{\eta}_{\sigma}^{\prime}=\vec{\chi}_{-\sigma}$, where $\psi=g^{1 / 2} \chi$ and $\eta=g^{-1 / 2} \chi$ and the result follows.

Remark. We have now shown in particular that the operator $\chi$ defined in connection with Lemma 4.3 is bounded in the case $\varepsilon=-$.

With the help of these lemmas we can deduce the factoriality of $\pi_{\omega} \mid D(\mathscr{C})$ in the case $\varepsilon=-$ by proving

Lemma 5.6. If $h \in H_{\sigma}^{-}(\mathscr{C}) \cap H_{-\sigma}^{-}(\mathscr{C})^{\prime}, \sigma= \pm \frac{1}{2}$, then $h=0$.

Proof. $h$ is the limit of a sequence $h_{n}$ with $h_{n} \in C_{0}^{\infty}(\mathscr{C})$. Pick $\chi \in C_{0}^{\infty}\left(\mathbb{R}^{3}\right)$ with $\chi(\vec{x})=1$ for $\vec{x} \in \mathscr{C}$ then, by Corollary 5.5, $\chi h_{n}$ converges in the $\|\cdot\|_{\sigma}^{0}$-topology to a $k \in H_{\sigma}^{0}(\mathscr{C})$. Now if $f \in C_{0}^{\infty}\left(\mathbb{R}^{3}\right)$

$$
\left(\omega^{-\sigma} f, \omega^{\sigma} h\right)^{-}=\lim \left(\omega^{-\sigma} f, \omega^{\sigma} \chi h_{n}\right)^{-}=\lim \left(\omega^{-\sigma} g^{1 / 2} f, \omega^{\sigma} \chi h_{n}\right)^{0}=\left(\omega^{-\sigma} g^{1 / 2} f, \omega^{\sigma} k\right)^{0} .
$$

Since $h$ has support in $\partial \mathscr{C}, k$ also has support in $\partial \mathscr{C}$. Thus $k=0$ and hence $h=0$.

We can therefore sum up the results of this section up to now as follows:

Theorem 5.7. If $\omega$ is an adiabatic vacuum state on an open Robertson-Walker spacetime then $\pi_{\omega} \uparrow \mathcal{O}$ is a factor when $\mathcal{O}=D(\mathscr{C})$ and $\mathscr{C}$ is an open bounded subset of some $\mathscr{S}_{t}$ with smooth boundary.

We conclude this section with some remarks on further properties of the nets of von Neumann algebras associated with adiabatic vacuum states. If $\mathcal{O}$ is an open subset of $\mathscr{M}$, we define $\mathfrak{R}_{S}(\mathcal{O})$ to be the von Neumann algebra generated by the Weyl operators $W(f)$ with $f=\Gamma f \in K(\mathcal{O})$ in the irreducible representation corresponding to the adiabatic vacuum state $\omega_{S}$. It is notationally convenient to interpret $\mathfrak{R}_{S}(X)$ for a general subset $X$ of $\mathscr{M}$ to be $\mathfrak{R}_{S}(\mathcal{O})$, where $\mathcal{O}$ is the interior of $X$. The net $\mathcal{O} \mapsto \mathfrak{R}_{S}(\mathcal{O})$ is trivially additive:

$$
\mathfrak{R}_{\mathrm{S}}(\mathcal{O})=\bigvee_{i} \mathfrak{R}_{\mathrm{S}}\left(\mathcal{O}_{i}\right), \quad \mathcal{O}=\bigcup_{i} \mathcal{O}_{i}
$$

where $\vee$ denotes the least upper bound in the lattice of von Neumann algebras. This follows from the fact that $\mathscr{D}(\mathscr{M})$ has partitions of the identity.

We adopt the analogous conventions for the net $\mathscr{C} \mapsto \mathfrak{R}_{S}(\mathscr{C})$ defined over the open subsets of some fixed $\mathscr{S}_{t}$. This net is again additive:

$$
\mathfrak{R}_{S}(\mathscr{C})=\bigvee_{i} \mathfrak{R}_{S}\left(\mathscr{C}_{i}\right), \quad \mathscr{C}=\bigcup_{i} \mathscr{C}_{i}
$$

We have also seen in Lemma 5.2 that

$$
\mathfrak{R}_{S}(\mathscr{C})=\mathfrak{R}_{S}(\mathscr{D}(\mathscr{C}))
$$

Now the phase space $(L, \Gamma, \gamma)$ and its local structure depend only on the differentiable manifold $\mathscr{S}_{t}$ and we have seen that, in the case $\varepsilon=0$, the topology corresponding to the scalar product $(\cdot,)_{S}$ is the same for all adiabatic vacuum states and all Robertson-Walker spacetimes with flat spatial sections. There are several properties of the net $\mathscr{C} \mapsto \mathfrak{R}_{S}(\mathscr{C})$ which are determined by $(L, \Gamma, \gamma)$ and this topology and are hence common to all adiabatic vacuum states including, of course, the Minkowski vacuum. A case in point is the factoriality of $\mathfrak{R}_{S}(\mathscr{C})$ discussed above. 
Another such property is duality: $\mathfrak{R}_{S}$ is said to satisfy duality for $\mathscr{C}$ if

$$
\mathfrak{R}_{S}(\mathscr{C})=\mathfrak{R}_{S}\left(\mathscr{C}^{c}\right)^{\prime},
$$

where $\mathscr{C}^{c}$ denotes the complement of $\mathscr{C}$. Again this property of the vacuum representation in Minkowski space was first proved by Araki [17] and we comment here on what is involved.

Duality is equivalent, cf. [4; Theorem 3.12], to

$$
L_{S}(\mathscr{C})=L_{S}\left(\mathscr{C}^{c}\right)^{\prime},
$$

and is thus, as claimed above, determined by $(L, \Gamma, \gamma)$ and the topology derived from $(\cdot,)_{S}$. Now $L_{S}\left(\mathscr{C}^{c}\right)^{\prime}$ consists of the elements of $L_{S}$ which have support in $\mathscr{C}^{-}$, the closure of $\mathscr{C}$. Next note that an element $F$ of $L_{S}$ with support in $\mathscr{C}$ is in $L_{S}(\mathscr{C})$ for the following reasons: we regard $\mathscr{S}_{t}$ as $\mathbb{R}^{3}$ and pick $k \in \mathscr{C}_{0}^{\infty}\left(\mathbb{R}^{3}\right)$ with $k(0)=1$ and set $k^{\varepsilon}(\vec{x})=k(\varepsilon \vec{x})$, then $k^{\varepsilon} F \rightarrow F$ in $L_{S}$ as $\varepsilon \rightarrow 0$, cf. [18; Theorem 2.2.11]. Hence we may assume that $F$ has compact support. Now pick $h \in \mathscr{C}_{0}^{\infty}\left(\mathbb{R}^{3}\right)$ with $\int d \vec{x} h=1$ and set $h_{\varepsilon}(\vec{x})=\varepsilon^{-3} h\left(\varepsilon^{-1} \vec{x}\right)$ then the convolution $h_{\varepsilon} * F$ converges to $F$ in $L_{S}$ as $\varepsilon \rightarrow 0$, c.f. [18; Theorem 2.2.10], and is $C^{\infty}$ with compact support in $\mathscr{C}$ for $\varepsilon$ sufficiently small thus $F \in L_{S}(\mathscr{C})$ as claimed. As a consequence we have

$$
L_{S}\left(\mathscr{C}^{c}\right)^{\prime}=\bigcap_{\mathscr{N}} L_{S}(\mathscr{C}+\mathscr{N})
$$

where $\mathscr{N}$ runs over the open neighbourhoods of the origin since an element of the right-hand side obviously has support in $\mathscr{C}^{-}$. Thus duality holds for $\mathscr{C}$ if and only if the net is outer regular at $\mathscr{C}$, i.e. if

$$
L_{S}(\mathscr{C})=\bigcap_{\mathscr{N}} L_{S}(\mathscr{C}+\mathscr{N})
$$

The discussion so far makes it clear that any remaining problem in proving duality for $\mathscr{C}$ can only concern the nature of the boundary of $\mathscr{C}$.

To prove duality for $\mathscr{C}$, the following assumption suffices: for each $\vec{x} \in \partial \mathscr{C}$ there is an open neighbourhood $U$ of $\vec{x}$ and a continuously differentiable function $\psi: U \rightarrow \mathbb{R}$ so that $\mathscr{C}^{-} \cap U=\{\vec{x} \in U: \psi(\vec{x}) \leqq 0\}$ and $\vec{\nabla} \psi(\vec{x}) \neq 0$ for all $\vec{x} \in U$. To see this let $F \in L_{S}$ have support in $\mathscr{C}^{-}$. As shown above, it suffices to suppose that $F$ has compact support and following Araki [17] we first prove

Lemma 5.8. Under the above assumptions on $\mathscr{C}$, there is for each $\vec{x} \in \mathscr{C}^{-}$an open ball $B\left(\vec{x}, \varepsilon_{\vec{x}}\right)$ centred on $\vec{x}$ with radius $\varepsilon_{\vec{x}}$ and a vector $\vec{n}_{\vec{x}}$ so that $\mathscr{C}^{-} \cap B\left(\vec{x}, \varepsilon_{\vec{x}}\right)+\lambda \vec{n}_{\vec{x}}$ has a positive distance from $\partial \mathscr{C}$ for all sufficiently small $\lambda>0$.

Proof. If $\vec{x} \in \mathscr{C}$, we can take $\vec{n}_{\vec{x}}=0$ and $\varepsilon_{\vec{x}}$ so small that $B\left(\vec{x}, \varepsilon_{\vec{x}}\right) \subset \mathscr{C}$. If $\vec{x} \in \partial \mathscr{C}$, we choose a $B\left(\vec{x}, \varepsilon_{1}\right)$ and a continuously differentiable function $\psi$ defined in a neighbourhood of $B\left(\vec{x}, \varepsilon_{1}\right)$ such that $B\left(\vec{x}, \varepsilon_{1}\right)^{-} \cap \mathscr{C}^{-}=\left\{\vec{x} \in B\left(\vec{x}, \varepsilon_{1}\right)^{-}: \psi(\vec{x}) \leqq 0\right\}$ and $\vec{\nabla} \psi(\vec{x}) \neq 0$ for $\vec{x} \in B\left(\vec{x}, \varepsilon_{1}\right)^{-}$. If we now pick $0<\varepsilon<\frac{1}{3} \varepsilon_{1}$ and translate $B(\vec{x}, \varepsilon)^{-} \cap \mathscr{C}^{-}$ by less than $\varepsilon$, the distance from the translated region to points of $\partial \mathscr{C}$ which lie outside $B\left(\vec{x}, \varepsilon_{1}\right)^{-}$is greater than $\frac{1}{3} \varepsilon_{1}$ so we have only to control the distance to $\partial \mathscr{C} \cap B\left(\vec{x}, \varepsilon_{1}\right)^{-}$. We translate in the direction of the inward pointing normal at $\vec{x}: \vec{n}_{\vec{x}}:=-\vec{\nabla} \psi(\vec{x}) /|\vec{\nabla} \psi(\vec{x})|$. Since $\vec{\nabla} \psi$ is continuous the Mean Value Theorem gives

$$
\psi\left(\vec{y}+\lambda \vec{n}_{\vec{x}}\right)=\psi(\vec{y})+\lambda \vec{n}_{\vec{x}} \cdot \vec{\nabla} \psi\left(\vec{y}+\vartheta \lambda \vec{n}_{\vec{x}}\right)
$$

for some $\vartheta \in[0,1]$ depending on $\vec{y}$. Furthermore since $\vec{\nabla} \psi(\vec{x}) \neq 0$, there is an $\varepsilon>0$ 
such that

$$
\left|\vec{\nabla} \psi(\vec{x})-\vec{\nabla} \psi\left(\vec{y}+\vartheta \lambda \vec{n}_{\vec{x}}\right)\right| \leqq \frac{1}{2}|\vec{\nabla} \psi(\vec{x})|
$$

for all $0<\lambda \leqq \varepsilon$ and all $\vec{y}$ with $|\vec{y}-\vec{x}| \leqq \varepsilon$. Hence

$$
\psi\left(\vec{y}+\lambda \vec{n}_{\vec{x}}\right) \leqq \psi(\vec{y})-\lambda|\vec{\nabla} \psi(\vec{x})|+\lambda\left|\vec{\nabla} \psi(\vec{x})-\vec{\nabla} \psi\left(\vec{y}+\vartheta \lambda \vec{n}_{\vec{x}}\right)\right| \leqq-\frac{1}{2} \lambda|\vec{\nabla} \psi(\vec{x})|<0
$$

for $0<\lambda<\varepsilon$ and all $\vec{y} \in \mathscr{C}^{-} \cap B(\vec{x}, \varepsilon)^{-}$. The compact sets $\mathscr{C}^{-} \cap B(\vec{x}, \varepsilon)^{-}+\lambda \vec{n}_{\vec{x}}$ and $\partial \mathscr{C} \cap B\left(\vec{x}, \varepsilon_{1}\right)^{-}=\left\{\vec{y} \in B\left(a, \varepsilon_{1}\right)^{-}: \psi(\vec{y})=0\right\}$ therefore have no common point and hence are separated by a strictly positive distance.

We now pick a finite open covering of $\operatorname{supp} F$ using the balls $B\left(\vec{x}, \varepsilon_{\vec{x}}\right)$ and a partition of the identity $\varphi_{v}, v=1,2, \ldots, n$, subordinate to this covering then $F=\sum_{v} \varphi_{v} F$, with $\varphi_{v} F \in L_{S}$. Hence it suffices to suppose that $F$ has support in $\mathscr{C}^{-} \cap B\left(\vec{x}, \varepsilon_{\vec{x}}\right)$. If we now translate $F$ through $\lambda \vec{n}_{\vec{x}}$ then $F_{\lambda_{\vec{n}}} \in L_{S}$ and has support in $\mathscr{C}$ for $0<\lambda<\varepsilon$. Hence $F_{\lambda \vec{n} \bar{x}} \in L_{S}(\mathscr{C})$. Hence letting $\lambda$ tend to zero and using the continuity of translations in $L_{S}$, we conclude that $F \in L_{S}(\mathscr{C})$. Hence we have proved duality (5.12) for all open sets $\mathscr{C}$ with $\mathscr{C}^{1}$-boundary.

We now pass to the case of the hyperbolic Robertson-Walker spacetime. $L_{S}\left(\mathscr{C}^{c}\right)^{\prime}$ again coincides with the distributions in $L_{S}$ with support in $\mathscr{C}^{-}$. To establish (5.12), we first show how distributions in $L_{S}$ can be approximated by distributions in $L_{S}$ with compact support.

Lemma 5.9. Regarding $\mathscr{S}^{-}$as $\mathbb{R}^{3}$ as before, let $\chi \in C_{0}^{\infty}\left(\mathbb{R}^{3}\right), 0 \leqq \chi \leqq 1$, with $\chi(\vec{x})=1$ for $|\vec{x}| \leqq 1$ and $\chi(\vec{x})=0$ for $|\vec{x}| \geqq 2$ and set $\chi^{\varepsilon}(\vec{x}):=\chi(\varepsilon \vec{x})$ for $\varepsilon \in(0,1]$ then the multiplication operators $\chi^{\varepsilon}$ on $L_{S}$ are uniformly bounded, i.e. $\left\|\chi^{\varepsilon}\right\|_{S} \leqq c, \varepsilon \in(0,1]$. If $F \in L_{S}$ then $\chi^{\varepsilon} F \in L_{S}$ has compact support and converges in $L_{S}$ to $F$ as $\varepsilon \rightarrow 0$.

Proof. We have to show that $\left\|\chi^{\varepsilon}\right\|_{\sigma} \leqq c$ for $\varepsilon \in(0,1]$ and $\sigma= \pm \frac{1}{2}$. However, if we prove this inequality for $\sigma=0,1$ it follows for $\sigma=\frac{1}{2}$ by interpolation theory [20; Chap. VIII] and for $\sigma=-\frac{1}{2}$ by dualizing. For $\sigma=0$ and $\sigma=1$, the estimates in Lemma 5.3 show that it suffices to prove that

$$
\begin{array}{r}
\sup _{\vec{x} \in \mathbb{R}^{3}}\left|\chi^{\varepsilon}(\vec{x})\right| \leqq c_{0}, \quad \varepsilon \in(0,1], \\
\sup _{\vec{x} \in \mathbb{R}^{3}} g^{i j}(\vec{x}) \partial_{i} \chi^{\varepsilon}(\vec{x}) \partial_{j} \chi^{\varepsilon}(\vec{x}) \leqq c_{1}, \quad \varepsilon \in(0,1] .
\end{array}
$$

Now (5.15) follows at once from the definition of $\chi^{\varepsilon}$. To prove (5.16), we note that in our coordinate system $g^{i j}(\vec{x})=x^{i} x^{j}$ for $i \neq j$ and $g^{i i}(\vec{x})=1+\left(x^{i}\right)^{2}$. Hence, we get

$$
\begin{aligned}
& \sup _{\vec{x} \in \mathbb{R}^{3}} g^{i j}(\vec{x}) \partial_{i} \chi^{\varepsilon}(\vec{x}) \partial_{j} \chi^{\varepsilon}(\vec{x}) \\
& \quad \leqq 9 \max _{i, j} \sup _{|\vec{x}| \leqq(2 / \varepsilon)} \varepsilon^{2}\left|g^{i j}(\vec{x})\right|\left|\partial_{i} \chi(\varepsilon \vec{x})\right|\left|\partial_{j} \chi(\varepsilon \vec{x})\right| \\
& \quad \leqq 9\left(\max _{i} \sup _{\vec{x}}\left|\partial_{i} \chi(\vec{x})\right|^{2}\right)\left(\sup _{|\vec{x}| \leqq(2 / \varepsilon)} \varepsilon^{2}\left(1+\vec{x}^{2}\right)\right) \leqq c^{\prime} \varepsilon^{2}\left(1+4 / \varepsilon^{2}\right) \leqq c_{1}
\end{aligned}
$$

Now, given $F \in L_{S}$, pick $F^{\prime} \in L=\mathscr{D}\left(\mathscr{S}^{-}\right) \times C_{0}^{\infty}\left(\mathscr{S}^{-}\right)$with $\left\|F^{\prime}-F\right\|_{S} \leqq \delta$ and $\varepsilon$ so small that $\chi^{\varepsilon} F^{\prime}=F^{\prime}$. Then $\left\|F-\chi^{\varepsilon} F\right\|_{S} \leqq\left\|F-F^{\prime}\right\|_{S}+\left\|\chi^{\varepsilon}\right\|_{S}\left\|F^{\prime}-F\right\|_{S} \leqq \delta(1+c)$, completing the proof. 
To complete the proof of duality for $\varepsilon=-$, we must show that distributions with compact support in $\mathscr{C}^{-}$can be approximated by elements of $L_{S}(\mathscr{C})$. Corollary 5.5. shows that the sets of distributions in $L_{S}$ with compact support for $\varepsilon=0$ and $\varepsilon=-$ agree. Let $F$ be a distribution with compact support in $\mathscr{C}^{-}$. Now we have already seen that there is a sequence of smooth functions $F_{n}$ with supports in a (bounded) subset of $\mathscr{C}$ which converge to $F$ in the $\|\cdot\|_{S}^{0}$-topology. Now by Corollary $5.5, F_{n}$ converges to $F$ in the $\|\cdot\|_{S}^{-}$-topology and we have proved duality for $\varepsilon=-$ for regions $\mathscr{C}$ with $C^{1}$-boundary.

\section{Appendix A}

In this appendix we summarize the relevant facts on the decomposition of the quasiregular representation $U$ of the groups $G^{\varepsilon}$ defined in Sect. 2 into a direct sum or direct integral of irreducible representations. In particular, we determine the commutant of $U\left(G^{-}\right)$.

The spherical harmonics $\mathscr{Y}_{\vec{k}}, \vec{k}=(k, l, m)(k=0,1, \ldots ; l=0,1, \ldots, k ; m=-l,-l+$ $1, \ldots, l)$, on the 3 -sphere form an orthonormal basis of eigenfunctions of the Laplace operator on $L^{2}\left(\mathscr{S}^{+}\right)$.

$$
\mathscr{Y}_{\vec{k}}(\psi, \vartheta, \varphi)=A_{k l} \Pi_{k l}^{+}(\psi) Y_{l, m}(\vartheta, \varphi),
$$

where the $Y_{l, m}$ are the usual spherical harmonics on the 2-sphere, $\Pi_{k l}^{+}(\psi)$ are real polynomials in $\sin \psi$ and $\cos \psi$ related to the Gegenbauer polynomials and $A_{k l}$ are real normalization constants. Since $\bar{Y}_{l, m}=Y_{l,-m}$, we have

$$
\overline{\mathscr{Y}}_{(k, l, m)}=\mathscr{Y}_{(k, l,-m)} \text {. }
$$

We let $\mathscr{H}_{k}$ denote the span of $\mathscr{Y}_{(k, l, m)}$ as $l$ and $m$ vary then the $\mathscr{H}_{k}$ are invariant subspaces and the restriction of $U\left(G^{+}\right)$to the $\mathscr{H}_{k}$ are pairwise equivalent irreducible representations. Our direct sum decomposition therefore takes the form

$$
L^{2}\left(\mathscr{S}^{+}\right)=\bigoplus_{k=0}^{\infty} \mathscr{H}_{k}
$$

In the case $\varepsilon=0$, we get a direct integral decomposition with the aid of a Fourier transform. To each $h \in C_{0}^{\infty}\left(\mathscr{S}^{0}\right)$ we associate a function $k \mapsto \widetilde{h}_{k}$ on $\mathbb{R}_{+}$, taking values in $L^{2}\left(S^{2}, d \Omega\right)$ :

$$
\tilde{h}_{k}(\vec{\xi}):=\int d \mu(\vec{x}) \mathscr{Y}_{k \vec{\xi}}(\vec{x}) h(\vec{x}),
$$

where $\mathscr{Y}_{\vec{k}}$ is given by (2.17). Since $\overline{\mathscr{Y}}_{\vec{k}}=\mathscr{Y}_{-\vec{k}}$, we have

$$
\widetilde{\widetilde{h}}_{k}(\vec{\xi})=\tilde{\bar{h}}_{k}(-\vec{\xi}) .
$$

The map $h \mapsto \tilde{h}$ extends to express $L^{2}\left(\mathscr{S}^{0}\right)$ as a direct integral of the constant field $L^{2}\left(S^{2}, d \Omega\right)$ over $\mathbb{R}_{+}$:

$$
L^{2}\left(\mathscr{S}^{0}\right)=\int^{\oplus} L^{2}\left(S^{2}, d \Omega\right) k^{2} d k
$$

There is an irreducible unitary representation $U_{k}$ of $G^{0}$ on $L^{2}\left(S^{2}, d \Omega\right)$ defined by

$$
U_{k}(g) \tilde{h}_{k}=\left(U(\widetilde{g) h})_{k}, \quad h \in C_{0}^{\infty}\left(\mathscr{S}^{0}\right),\right.
$$


and we get a decomposition of $U$ into a direct integral of equivalent irreducible representations.

If we replace $\mathscr{S}^{0}$ by $\mathscr{S}^{-}$and define $\mathscr{Y}_{\vec{k}}$ by (2.19) then (A.3), (A.5) and (A.6) yield us a direct integral decomposition of the representation $U$ of $G^{-}$into inequivalent irreducible unitary representations [10; Chap. VI, Sect. 3.3]. We will now show that

$$
\int d \Omega(\vec{\xi}) \overline{\tilde{f}}_{k}(\vec{\xi}) \tilde{h}_{k}(\vec{\xi})=\int d \Omega(\vec{\xi}) \tilde{\bar{f}}_{k}(\vec{\xi}) \overline{\tilde{\bar{h}}}_{k}(\vec{\xi}), \quad f, h \in C_{0}^{\infty}(\mathscr{S}) .
$$

When $\varepsilon=0$ this equation follows from (A.4) and when $\varepsilon=+$ it follows from (A.2) provided we interpret the integration as a sum over the discrete variables $l$ and $m$. When $\varepsilon=-$, we consider a Lorentz trasformation $L_{x y}$ which interchanges two points $x, y \in \mathscr{S}^{-}: L_{x y}(x)=y, L_{x y}(y)=x$. Given $f, h \in C_{0}^{\infty}\left(\mathscr{S}^{-}\right)$we have

$$
\begin{aligned}
\int d \Omega(\vec{\xi}) \overline{\tilde{f}}_{k}(\vec{\xi}) \tilde{h}_{k}(\vec{\xi}) & =(2 \pi)^{-3} \int d \Omega(\vec{\xi}) \int d \mu(\vec{x}) \int d \mu(\vec{y})(x \cdot \xi)^{-1+i k} \bar{f}(\vec{x})(y \cdot \xi)^{-1-i k} h(\vec{y}) \\
& =(2 \pi)^{-3} \int d \mu(\vec{x}) d \mu(\vec{y}) \int d \Omega(\vec{\xi})\left(y \cdot L_{x y}^{-1} \xi\right)^{-1+i k} \bar{f}(\vec{x})\left(x \cdot L_{x y}^{-1} \xi\right)^{-1-i k} h(\vec{y}) \\
& =(2 \pi)^{-3} \int d \mu(\vec{x}) d \mu(\vec{y}) \int d \Omega(\vec{\xi})(y \cdot \xi)^{-1+i k} \bar{f}(\vec{x})(x \cdot \xi)^{-1-i k} h(\vec{y}) \\
& =\int d \Omega(\vec{\xi}) \tilde{\tilde{f}}_{k}(\vec{\xi}) \overline{\tilde{h}}_{k}(\vec{\xi}) .
\end{aligned}
$$

The third equality here rests on the fact that $U_{k}\left(L_{x y}\right)$ is a unitary operator (cf. [11; Chap. X, Sect. 2, Sect. 4]).

We shall also have occasion to use the inverse Fourier transform given by

$$
h(\vec{x})=\int d \vec{k} \mathscr{Y}_{\vec{k}}(\vec{x}) \tilde{h}(\vec{k}),
$$

(cf. [10; Chap. VI, Sect. 3.3]).

The main result of this appendix is the following:

Lemma A.1. The commutant of $U\left(G^{-}\right)$consists of the diagonalizable operators, i.e. of the operators $T$ of the form

$$
(\widetilde{T f})_{k}=t(k) \tilde{f}_{k}
$$

where $k \mapsto t(k) \in \mathbb{C}$ is an essentially bounded, measurable function.

Proof. We use [12; Proposition 8.6.4] and refer to [21] for statements about the spectrum of the group $S L(2, \mathbb{C})$. The above direct integral decomposition gives us a measurable field $k \mapsto U_{k}$ of continuous unitary representation of $\mathscr{L}_{+}^{\dagger}(4)$, and hence of $S L(2, \mathbb{C})$, on the standard Borel space $\mathbb{R}_{+}$. Let $\pi$ and $\tilde{\pi}_{k}$ be the representations of $C^{*}(S L(2, \mathbb{C}))$, the $C^{*}$-algebra of the group, associated with $U$ and $U_{k}$ respectively, then

$$
\pi=\int^{\oplus} \tilde{\pi}_{k} k^{2} d k, \quad U(S L(2, \mathbb{C}))^{\prime}=\pi\left(C^{*}(S L(2, \mathbb{C}))\right)^{\prime}
$$

$C^{*}(S L(2, \mathbb{C}))$ is a liminal $C^{*}$-algebra $[22]$ and is separable since $S L(2, \mathbb{C})$ is separable. The spectrum of $C^{*}(S L(2, \mathbb{C}))$ is therefore a standard Borel space [12; 8.6.1]. It can be considered as a subset of $\mathbb{R}^{2}$ which carries the induced topology with the exception of two points [21]. In particular, the mapping $\eta: k \in \mathbb{R}_{+} \mapsto\left[\tilde{\pi}_{k}\right]$, the equivalence class of $\tilde{\pi}_{k}$, is a continuous injection and a Borel isomorphism of $\mathbb{R}_{+}$ and $\eta\left(\mathbb{R}_{+}\right)$. Now let $\xi \mapsto \pi_{\xi}$ be the canonical field of representations on the spectrum of $C^{*}(S L(2, \mathbb{C}))$ as in [12; Proposition 8.6.4] and $\hat{\pi}:=\int^{\oplus} \pi_{\xi} d v(\xi)$, where $v$ is the image of $k^{2} d k$ under $\eta$. Then $\pi_{\eta(k)}$ is unitarily equivalent to $\tilde{\pi}_{k}$ and, by [12; Proposition 
8.2.3], there is a unitary operator $V: \mathscr{H}_{\pi} \rightarrow \mathscr{H}_{\hat{\pi}}$ with $\hat{\pi}(A)=V \pi(A) V^{*}, A \in C^{*}(S L(2, \mathbb{C}))$ and $\hat{Z}=V Z V^{*}$, where $Z$ and $\hat{Z}$ denote the algebra of diagonalizable operators of $\mathscr{H}_{\pi}$ and $\mathscr{H}_{A}$ respectively. Since by $[12$; Proposition 8.6 .4$] \hat{\pi}\left(C^{*}(S L(2, \mathbb{C}))\right)^{\prime}=\hat{Z}$, $\pi\left(C^{*}(S L(2, \mathbb{C}))\right)^{\prime}=Z$, as required.

\section{Appendix B}

In this appendix we deduce using standard arguments the results quoted in Sect. 3 on the solution of the coupled integral equations (3.15) and (3.16). form

We use matrix notation writing $x=\left(\begin{array}{l}\alpha \\ \beta\end{array}\right)$ so that our equations have the general

$$
x=y+T x
$$

where $y=\left(\begin{array}{l}1 \\ 0\end{array}\right),(T x)(t)=\int_{t_{1}}^{t} T\left(t^{\prime}\right) x\left(t^{\prime}\right) d t^{\prime}$ and $T\left(t^{\prime}\right)$ is the $2 \times 2$-matrix derived from (3.15) and (3.16) with $S$ being given by (3.18). We work over a fixed compact interval of time $I$. There is also an implicit $k$-dependence, $x=x_{k}$ and $T=T_{k}$ and by Lemma 3.2 , there is a $C>0$ such that

$$
\left|T_{k}^{i j}(t)\right| \leqq C(1+k)^{-2 n-1}, \quad t \in I .
$$

For each fixed $k$, we look for solutions of (B.1) where the components of $x$ are supposed to be continuous functions of $t \in I$ and we work in the Banach space obtained by using a norm adapted to the problem:

$$
\|x\|_{w}:=\max _{i=1,2} \sup _{t \in I}\left|\frac{x^{i}(t)}{w(t)}\right|
$$

where

$$
w(t):=e^{L\left|t-t_{1}\right|}, \quad L \geqq 4 C .
$$

We check using (B.2) that $T$ is contractive:

$$
\|T x\|_{w} \leqq \frac{2 C}{L}(1+k)^{-2 n-1}\|x\|_{w} \leqq \frac{1}{2}\|x\|_{w} .
$$

The unique solution to (B.1) is therefore given by

$$
x=\sum_{n=0}^{\infty} T^{n} y .
$$

We now investigate the dependence of the solution on the parameter $k$. For two solutions $x_{k}, x_{k^{\prime}}$ we have

$$
\begin{aligned}
\left\|x_{k}-x_{k^{\prime}}\right\|_{w} & \leqq \sum_{n=1}^{\infty}\left\|\left(T_{k}^{n}-T_{k^{\prime}}^{n}\right) y\right\|_{w} \leqq \sum_{n=1}^{\infty} \sum_{v=1}^{n}\left\|T_{k}^{v-1}\left(T_{k}-T_{k^{\prime}}\right) T_{k^{\prime}}^{n-v}\right\|_{w}\|y\|_{w} \\
& \leqq 2 \sum_{n=1}^{\infty} \frac{n}{2^{n}}\left\|T_{k}-T_{k^{\prime}}\right\|_{w}\|y\|_{w} \leqq \widetilde{C}\left\|T_{k}-T_{k^{\prime}}\right\|_{w}
\end{aligned}
$$


and

$$
\left\|T_{k}-T_{k^{\prime}}\right\|_{w} \leqq \frac{2}{L} \max _{i, j}\left\{\sup _{t \in I}\left|T_{k}^{i j}(t)-T_{k^{\prime}}^{i j}(t)\right|\right\} .
$$

Since by Lemmas 3.2 and (3.18), $(k, t) \mapsto T_{k}^{i j}(t)$ is uniformly continuous on compacta, we deduce that $k \mapsto x_{k}(t)$ is continuous uniformly in $t \in I$.

To deduce that $\beta_{k}=O\left(k^{-2 n-1}\right)$ uniformly in $t \in I$, we proceed as follows:

$$
\sup _{t \in I}\left|\frac{\beta_{k}(t)}{w(t)}\right| \leqq\left\|x_{k}-y\right\|_{w} \leqq \sum_{n=0}^{\infty}\left\|T_{k}\right\|_{w}^{n}\left\|T_{k} y\right\|_{w} \leqq \frac{4 C}{L}(1+k)^{-2 n-1}\|y\|_{w},
$$

where we have made use of (B.5). Thus

$$
\left|\beta_{k}(t)\right| \leqq(1+k)^{-2 n-1}\|y\|_{w} \sup _{t \in I} w(t)
$$

as required.

\section{Appendix C}

In showing that the distribution $G(x, y)$ defined by (2.42) yields the operator $E$ via (2.44), we were forced to interchange differentiation and integration in a few places. This step will be justified in this appendix and we shall need some properties of the function $G_{k}\left(x^{0}, y^{0}\right)$ defined by (2.43) using solutions $T_{k}$ of the differential equation (2.40) which satisfy the subsidiary condition (2.41). These properties are more easily derived if, as in Sect. 3, we replace the differential equation (2.40) by two equivalent first order differential equations, which can then be studied using the methods of Appendix B.

As we remarked preceding Lemma $2.4, G_{k}$ is independent of the choice of solutions $T_{k}$. Thus, we can, for example, use the solution with initial conditions

$$
T_{k}\left(t_{1}\right)=W_{k}\left(t_{1}\right), \quad \dot{T}_{k}\left(t_{1}\right)=\dot{W}_{k}\left(t_{1}\right),
$$

where $W_{k}(t):=W_{k}^{(0)}(t)$ is given by (3.5). If we write

$$
T_{k}(t)=\alpha_{k}(t) W_{k}(t)+\beta_{k}(t) \bar{W}_{k}(t),
$$

where $\alpha_{k}$ and $\beta_{k}$ are solutions of the integral equations (3.15) and (3.16) with $\Omega_{k}=\Omega_{k}^{(0)}$ then we see after differentiating and some manipulation that $T_{k}$ satisfies the differential equation (2.40) with initial conditions given by (C.1) and we have

$$
\dot{T}_{k}=\alpha_{k} \dot{W}_{k}+\beta_{k} \dot{\bar{W}}_{k} \text {. }
$$

Conversely, given a solution of (2.40) of the form (C.2) satisfying (C.1) and (C.3), we get, using (3.14), a solution of the integral equations (3.15) and (3.16).

Now the methods of Appendix B yield, for each compact interval $I \subset \mathbb{R}$, a unique continuous solution $t \mapsto\left(\alpha_{k}(t), \beta_{k}(t)\right)$ of (3.15) and (3.16) and $\alpha_{k}=O(1)$ and $\beta_{k}=O\left(k^{-1}\right)$ each uniformly for $t \in I$ and $k \mapsto\left(\alpha_{k}(t), \beta_{k}(t)\right)$ is uniformly continuous for $t \in I$. In particular $(k, t) \mapsto\left(\alpha_{k}(t), \beta_{k}(t)\right)$ is continuous. Furthermore, being solutions of (3.15) and (3.16), $\alpha_{k}$ and $\beta_{k}$ will actually be continuously differentiable in $t$. It now follows from (C.2) and (C.3) that the mapping $(k, t) \mapsto\left(T_{k}(t), \dot{T}_{k}(t)\right)$ is continuous and $T_{k}=O\left(k^{-1 / 2}\right), \dot{T}_{k}=O\left(k^{1 / 2}\right)$ uniformly in $t \in I$. Using the differential equation 
(2.40) one can prove by induction that $(k, t) \mapsto T_{k}^{(v)}(t)$ is continuous and

$$
T_{k}^{(v)}=O\left(k^{v-1 / 2}\right)
$$

uniformly in $t \in I$, where $T_{k}^{(v)}$ denotes the $v^{\text {th }}$ derivative of $T_{k}$.

In addition to these properties of solutions of (2.40) we need estimates on the derivatives of the $\mathscr{Y}_{\vec{k}}$. Since on any compact set $K$ of $\mathscr{S}^{-}$we have

$$
(x \cdot \xi)=\left(1+\vec{x}^{2}\right)^{1 / 2}-\vec{\xi} \cdot \vec{x} \geqq\left(1+\vec{x}^{2}\right)^{1 / 2}-|\vec{x}| \geqq c>0,
$$

we see immediately that

$$
\sup _{K}\left|D^{v} \mathscr{Y}_{\vec{k}}\right| \leqq C(v ; K)(1+k)^{|v|} .
$$

In the case $\varepsilon=+$, there is a similar inequality in [23]. $\mathscr{S}^{+}$is regarded as in (2.2) as being imbedded in $\mathbb{R}^{4}$ and $\mathscr{Y}_{\vec{k}}$ is regarded as a function of $x=\left(x^{0}, x^{1}, x^{2}, x^{3}\right)$. $P_{\vec{k}}(x):=|x|^{k} \mathscr{Y}_{\vec{k}}(x)$ is then a harmonic polynomial and using this fact it is proved in Appendix $\mathrm{C}$ of [23] that

$$
\sup _{|x|=1}\left|D^{v} \mathscr{Y}_{\vec{k}}\right| \leqq C(v) k^{2+|v|}, \quad k \neq 0 .
$$

Now given $f \in \mathscr{D}(\mathscr{M})$, choose an $I \subset \mathbb{R}$ such that $x^{0}$ and $\left\{y^{0}: f\left(y^{0}, \vec{y}\right) \neq 0\right\}$ are in the interior of $I$. Let $T_{k}$ be the solution of (2.40) with (C.1) as initial conditions. To show that the order of differentiation and integration can be exchanged in the expression for $G(x, f)$ from (2.44) we must show that

$$
\gamma\left(x, y^{0}, \vec{k}\right):=\mathscr{Y}_{\vec{k}}(\vec{x}) G_{k}\left(x^{0}, y^{0}\right) \check{f}\left(y^{0}, \vec{k}\right)
$$

and each of its derivatives with respect to $x$ can be majorized by integrable functions.

The continuity of the derivatives of $\gamma$ with respect to $x$ follows from the same property of $\mathscr{Y}_{\vec{k}}$ and $G_{k}\left(\cdot, y^{0}\right)$. From (C.4)-(C.6) we get

$$
\sup _{x \in I \times K}\left|\left(D_{x}^{v} \gamma\right)\left(x, y^{0}, \vec{k}\right)\right| \leqq C_{v}(I, K)(1+k)^{2+|v|}\left|\breve{f}\left(y^{0}, \vec{k}\right)\right|
$$

and

$$
\begin{aligned}
& \int d y^{0} \int d \vec{k}(1+k)^{2+|v|}\left|\breve{f}\left(y^{0}, \vec{k}\right)\right| \\
& \quad \leqq\left(\int d \vec{k}\left|\frac{(1+k)^{2+|v|}}{(1+E(k))^{m}}\right|^{2}\right)^{1 / 2} \int d y^{0}\left(\int d \vec{k}\left|(1+E(k))^{m} \check{f}\left(y^{0}, \vec{k}\right)\right|^{2}\right)^{1 / 2} \\
& \quad \leqq c \int d y^{0}\left(\int\left|\left((1-\Delta)^{m} f\right)\left(y^{0}, \vec{y}\right)\right|^{2} d \mu(\vec{y})\right)^{1 / 2}<+\infty
\end{aligned}
$$

for $m \in \mathbb{N}, m>\frac{1}{2}\left(\frac{7}{2}+|v|\right)$. The Dominated Convergence Theorem now shows that we can interchange differentiation and integration.

\section{Appendix D}

In this appendix we use the method of stationary phase to prove (4.17). Since the continuous function $\left(\vec{k}, \vec{k}^{\prime}\right) \mapsto \hat{\chi}\left(\vec{k}, \vec{k}^{\prime}\right)$ is bounded, it suffices to show that for each $N \in \mathbb{N}_{0}$ there is a $c_{N}>0$ such that

$$
\left|\hat{\chi}\left(\vec{k}, \vec{k}^{\prime}\right)\right| \leqq c_{N}\left|\vec{k}-\vec{k}^{\prime}\right|^{-N}, \quad \vec{k} \neq \vec{k}^{\prime}
$$


To this end, we write $\hat{\chi}$ in the form

$$
\hat{\chi}\left(\vec{k}, \vec{k}^{\prime}\right)=\int d \vec{x} u\left(\vec{x} ; \vec{\xi}, \vec{\xi}^{\prime}\right) e^{i\left|\vec{k}-\vec{k}^{\prime}\right| f\left(\vec{x} ; \vec{k}, \vec{k}^{\prime}\right)}
$$

where

and

$$
u\left(\vec{x} ; \vec{\xi}, \vec{\xi}^{\prime}\right):=(2 \pi)^{-3}(x \cdot \xi)^{-1} \chi(\vec{x})\left(x \cdot \xi^{\prime}\right)^{-1} g^{1 / 2}(\vec{x})
$$

$$
f\left(\vec{x} ; \vec{k}, \vec{k}^{\prime}\right):=\left|\vec{k}-\vec{k}^{\prime}\right|^{-1}\left\{k^{\prime} \ln \left[\left(1+\vec{x}^{2}\right)^{1 / 2}-\vec{\xi}^{\prime} \cdot \vec{x}\right]-k \ln \left[\left(1+\vec{x}^{2}\right)^{1 / 2}-\vec{\xi} \cdot \vec{x}\right]\right\} .
$$

To prove (D.1) using [16; Theorem 7.7.1], we must show that for all multi-indices $\alpha$,

$$
\begin{array}{ll}
\left|\left(D_{x}^{\alpha} u\right)\left(\vec{x} ; \vec{\xi}, \vec{\xi}^{\prime}\right)\right| \leqq c_{\alpha}, \quad \vec{\xi}, \vec{\xi}^{\prime} \in S^{2}, \quad \vec{x} \in \mathbb{R}^{3}, \\
\left|\left(D_{x}^{\alpha} f\right)\left(\vec{x} ; \vec{k}, \vec{k}^{\prime}\right)\right| \leqq c_{\alpha}^{\prime}, \quad \vec{k}, \vec{k}^{\prime} \in \mathbb{R}^{3}, \quad \vec{x} \in \operatorname{supp} \chi, \\
\left|\left(\vec{\nabla}_{x} f\right)\left(\vec{x} ; \vec{k}, \vec{k}^{\prime}\right)\right| \geqq c>0, \quad \vec{k}, \vec{k}^{\prime} \in \mathbb{R}^{3}, \quad \vec{x} \in \operatorname{supp} \chi .
\end{array}
$$

The following lemma yields (D.7) only if supp $\chi \subset \mathscr{B} \subset \mathscr{S}$, where $\mathscr{B}:=\left\{x \in \mathscr{S}:|\vec{x}|<\frac{1}{3}\right\}$. To prove (D.1) for a general $\chi \in C_{0}^{\infty}(\mathscr{S})$, we make use of the fact that $\mathscr{S}$ is a homogeneous space under Lorentz transformations.

Lemma D.1. Let $\chi \in C_{0}^{\infty}(\mathscr{B})$, then $(D .5),(D .6)$ and (D.7) hold and hence (D.1) holds, too. Proof. Since $\chi \in C_{0}^{\infty}(\mathscr{B})$ and $(x \cdot \xi)$ is strictly positive on each compact set, (D.5) follows immediately. Now we have

$$
\begin{aligned}
(\nabla f)_{i}= & h\left(\vec{x} ; \xi, \xi^{\prime}\right)\left|\vec{k}-\vec{k}^{\prime}\right|^{-1} \\
& \cdot\left\{\frac{x_{i}}{\left(1+\vec{x}^{2}\right)^{1 / 2}}\left(k^{\prime}(x \cdot \xi)-k\left(x \cdot \xi^{\prime}\right)\right)+k \xi_{i}\left(x \cdot \xi^{\prime}\right)-k^{\prime} \xi_{i}^{\prime}(x \cdot \xi)\right\}, \\
h\left(\vec{x} ; \xi, \xi^{\prime}\right):= & (x \cdot \xi)^{-1}\left(x \cdot \xi^{\prime}\right)^{-1} .
\end{aligned}
$$

$h$ is a smooth function in $\vec{x}$. The term in curly brackets and its derivatives with respect to $\vec{x}$ are sums of terms of the form $k^{\prime}-k, k \xi_{i}-k^{\prime} \xi_{i}^{\prime}, k^{\prime} \xi_{j}-k \xi_{j}^{\prime}$ or $k \xi_{i} \xi_{j}^{\prime}-k^{\prime} \xi_{i}^{\prime} \xi_{j}$ times a smooth function of $\vec{x}$. These terms are bounded by an expression of the form $A\left|\vec{k}-\vec{k}^{\prime}\right|$, for example

$$
\left|k \xi_{i} \xi_{j}^{\prime}-k^{\prime} \xi_{i}^{\prime} \xi_{j}\right| \leqq\left|k_{i} \xi_{j}^{\prime}-k_{i}^{\prime} \xi_{j}^{\prime}\right|+\left|\xi_{i}^{\prime} k_{j}^{\prime}-\xi_{i}^{\prime} k_{j}\right|+\left|k \xi_{i}^{\prime} \xi_{j}-k^{\prime} \xi_{i}^{\prime} \xi_{j}\right| \leqq 3\left|\vec{k}-\vec{k}^{\prime}\right| .
$$

This yields (D.6) for $\alpha \neq 0$. One gets (D.6) for $\alpha=0$ from the estimate for $\vec{\nabla} f$ using

$$
f\left(\vec{x} ; \vec{k}, \vec{k}^{\prime}\right)=\int_{0}^{1}(\vec{x} \cdot \vec{\nabla} f)\left(t \vec{x} ; \vec{k}, \vec{k}^{\prime}\right) d t .
$$

To prove (D.7), we note that using $\left|\vec{k}\left(x \cdot \xi^{\prime}\right)-\vec{k}^{\prime}(x \cdot \xi)\right| \geqq\left|k\left(x \cdot \xi^{\prime}\right)-k^{\prime}(x \cdot \xi)\right|$ we have

$$
|\vec{\nabla} f| \geqq\left[\left(1+\vec{x}^{2}\right)^{1 / 2}+|\vec{x}|\right]^{-2}\left|\vec{k}-\vec{k}^{\prime}\right|^{-1}\left[1-\frac{|\vec{x}|}{\left(1+\vec{x}^{2}\right)^{1 / 2}}\right]\left|\vec{k}\left(x \cdot \xi^{\prime}\right)-\vec{k}^{\prime}(x \cdot \xi)\right| .
$$

However

$$
\begin{aligned}
& \left|\vec{k}\left(x \cdot \xi^{\prime}\right)-\vec{k}^{\prime}(x \cdot \xi)\right| \geqq\left|\vec{k}-\vec{k}^{\prime}\right|\left(1+\vec{x}^{2}\right)^{1 / 2}-\left|\vec{k}\left(\vec{x} \cdot \vec{\xi}^{\prime}\right)-\vec{k}^{\prime}(\vec{x} \cdot \vec{\xi})\right| \\
& \quad \geqq\left[\left(1+\vec{x}^{2}\right)^{1 / 2}-3|\vec{x}|\right]\left|\vec{k}-\vec{k}^{\prime}\right|,
\end{aligned}
$$


where the second inequality is proved similarly to (D.8). We therefore have $|\vec{\nabla} f| \geqq c$ for $|\vec{x}| \leqq \frac{1}{3}$ and we have proved (D.7) completing the proof of the lemma.

We now want to prove (D.1) for an arbitrary $\psi \in C_{0}^{\infty}(\mathscr{S})$. In fact, it suffices to show that if $\chi \in C_{0}^{\infty}(\mathscr{B})$ and $\Lambda$ is a Lorentz transformation then $\chi_{\Lambda}$ satisfies (D.1), where $\chi_{\Lambda}(\vec{x}):=\chi\left(\Lambda^{-1}(\vec{x})\right)$ and $\vec{x}$ coordinatizes the point $x=\left(\left(1+\vec{x}^{2}\right)^{1 / 2}, \vec{x}\right)$ on the hyperboloid $\mathscr{S}^{-}$in Minkowski space. To see this, note that supp $\psi \subset \bigcup_{\Lambda} \Lambda \mathscr{B}$ and picking a finite subcovering and a smooth partition of the identity subordinate to this subcovering $\psi$ is just a finite sum of functions of the form $\chi_{A}$ with $\chi \in C_{0}^{\infty}(\mathscr{B})$. Furthermore, it suffices to prove (D.1) for the case that $\Lambda$ is a boost in the $x^{1}$-direction since every Lorentz transformation is a product of rotations and such a boost and it follows from the form of the eigenfunctions $\mathscr{Y}_{\vec{k}}$ of (2.19) that if $\chi$ satisfies (D.1) then so does the rotated $\chi$. For such a boost, we have

$$
\begin{aligned}
\hat{\chi}_{\Lambda}\left(\vec{k}, \vec{k}^{\prime}\right) & =\left(\operatorname{ch} \alpha-\xi^{1} \operatorname{sh} \alpha\right)^{-1-i k}\left(\operatorname{ch} \alpha-\xi^{1} \operatorname{sh} \alpha\right)^{-1+i k^{\prime}} \hat{\chi}\left(k \Lambda^{-1}(\vec{\xi}), k^{\prime} \Lambda^{-1}\left(\vec{\xi}^{\prime}\right)\right), \\
\Lambda^{-1}(\vec{\xi}) & =n(\alpha, \vec{\xi})^{-1}\left(\xi^{1} \operatorname{ch} \alpha-\operatorname{sh} \alpha, \xi^{2}, \xi^{3}\right) \in S^{2}, \quad n(\alpha, \vec{\xi})=\operatorname{ch} \alpha-\xi^{1} \operatorname{sh} \alpha .
\end{aligned}
$$

Hence, as $\left|\xi^{1}\right| \leqq|\vec{\xi}|=1$, ch $\alpha-\xi^{1} \operatorname{sh} \alpha>\delta>0$, and we must show

$$
\left|\hat{\chi}\left(k \Lambda^{-1}(\vec{\xi}), k^{\prime} \Lambda^{-1}\left(\vec{\xi}^{\prime}\right)\right)\right| \leqq c_{N}\left|\vec{k}-\vec{k}^{\prime}\right|^{-N}
$$

or, equivalently that

$$
\left|\hat{\chi}\left(k \vec{\xi}, k^{\prime} \vec{\xi}^{\prime}\right)\right| \leqq c_{N}\left|k \Lambda(\vec{\xi})-k^{\prime} \Lambda\left(\vec{\xi}^{\prime}\right)\right|^{-N} .
$$

Since $\chi$ itself satisfies (D.1), it suffices to show that

$$
\left|k \Lambda(\vec{\xi})-k^{\prime} \Lambda\left(\vec{\xi}^{\prime}\right)\right| \leqq c\left|\vec{k}-\vec{k}^{\prime}\right|
$$

Now

$$
\Lambda(\vec{\xi})=n^{-1}\left(\xi^{1} \operatorname{ch} \alpha+\operatorname{sh} \alpha, \xi^{2}, \xi^{3}\right), \quad n=n(-\alpha, \vec{\xi})
$$

and have

$$
\begin{aligned}
\left|k \Lambda(\vec{\xi})-k^{\prime} \Lambda\left(\vec{\xi}^{\prime}\right)\right| \leqq & \left(n n^{\prime}\right)^{-1}\left\{\left|n^{\prime} \vec{k}-n \vec{k}^{\prime}\right|+\mid n^{\prime} k\left[\xi^{1}(\operatorname{ch} \alpha-1)+\operatorname{sh} \alpha\right]\right. \\
& \left.-n k^{\prime}\left[\xi^{\prime 1}(\operatorname{ch} \alpha-1)+\operatorname{sh} \alpha\right] \mid\right\},
\end{aligned}
$$

where $n^{\prime}=n\left(-\alpha, \vec{\xi}^{\prime}\right)$. The first term in this expression may be estimated as follows:

$$
\left|n^{\prime}\left(\vec{k}-\vec{k}^{\prime}\right)+\operatorname{sh} \alpha \vec{\xi}^{\prime}\left(k^{\prime} \xi^{\prime 1}-k \xi^{1}\right)+\xi^{1} \operatorname{sh} \alpha \vec{\xi}^{\prime}\left(k-k^{\prime}\right)\right| \leqq\left(n^{\prime}+2|\operatorname{sh} \alpha|\right)\left|\vec{k}-\vec{k}^{\prime}\right| .
$$

The second term can be estimated by

$$
n^{\prime}\left|\operatorname{sh} \alpha\left(k-k^{\prime}\right)+(\operatorname{ch} \alpha-1)\left(k \xi^{1}-k^{\prime} \xi^{\prime 1}\right)\right|+\left|\left(n^{\prime}-n\right) k^{\prime}\left[\xi^{\prime 1}(\operatorname{ch} \alpha-1)+\operatorname{sh} \alpha\right]\right|,
$$

and arguing as above we have

$$
\left|\left(n^{\prime}-n\right) k^{\prime}\right| \leqq 2|\operatorname{sh} \alpha|\left|\vec{k}-\vec{k}^{\prime}\right| .
$$

From these estimates we deduce (D.10) and hence (D.1) for all $\chi \in C_{0}^{\infty}(\mathscr{S})$. 


\section{References}

1. Haag, R., Narnhofer, H., Stein, U.: On quantum field theory in gravitational background. Commun. Math. Phys. 94, 219-238 (1984)

2. Haag, R., Kastler, D.: An algebraic approach to quantum field theory. J. Math. Phys. 5, 848-861 (1964)

3. Dimock, J.: Algebras of local observables on a manifold. Commun. Math. Phys. 77, 219-228 (1980)

4. Araki, H., Yamagami, S.: On quasiequivalence of quasifree states of the canonical commutation relations. Publ. RIMS, Kyoto 18, 283-338 (1982)

5. Najmi, A. H., Ottewill, A. C.: Quantum states and the Hadamard form. III. Constraints in cosmological space-times. Phys. Rev. D32, 1942-1948 (1985)

6. Bernard, D.: Hadamard singularity and quantum states in Bianchi type-I space-time. Phys. Rev. D33, 3581-3589 (1986)

7. Mazzitelli, F. D., Paz, J. P., Castagnino, M. A.: Cauchy data and Hadamard singularities in time-dependent backgrounds. Phys. Rev. D36, 2994-3001 (1987)

8. Fulling, S. A., Narcowich, F. J., Wald, R. M.: Singularity structure of the two-point function in quantum field theory in curved spacetime II. Ann. Phys. 136, 243-272 (1981)

9. Kay, B. S.: Linear spin-zero quantum fields in external gravitational and scalar fields $I$. Commun. Math. Phys. 62, 55-70 (1978)

10. Gelfand, I. M., Graev, M. I., Vilenkin, N. J.: Generalized functions, vol. 5. New York, London: Academic Press 1966

11. Vilenkin, N. J.: Special functions and the theory of group representations. Providence, Rhode Island: American Mathematical Society 1968

12. Dixmier, J.: $C^{*}$-Algebras. Amsterdam, New York, Oxford: North-Holland 1977

13. Parker, L.: Quantized fields and particle creation in expanding universes I. Phys. Rev. 183, $1057-1068$ (1969)

14. Parker, L., Fulling, S. A.: Adiabatic regularization of the energy-momentum tensor of a quantized field in homogeneous spaces. Phys. Rev. D9, 341 -354 (1974)

15. Buchholz, D.: Product states for local algebras. Commun. Math. Phys. 36, 287-304 (1974)

16. Hörmander, L.: The analysis of linear partial differential operators I. Berlin, Heidelberg, New York: Springer 1983

17. Araki, H.: Von Neumann algebras of local observables for the free scalar field. J. Math. Phys. 5, 1-13 (1964)

18. Hörmander, L.: Linear partial differential operators. Berlin, Heidelberg, New York: Springer 1976

19. Trèves, F.: Basic linear partial differential equations. New York, San Francisco, London: Academic Press 1975

20. Palais, R. S.: Seminar on the Atiyah-Singer index theorem. Princeton, New Jersey: Princeton University Press 1965

21. Fell, J. M. G.: The dual spaces of $C^{*}$-algebras. Trans. Am. Math. Soc. 94, 365-403 (1960)

22. Fell, J. M. G.: The structure of algebras of operator fields. Acta Math. 106, 233-280 (1961)

23. Stein, E. M.: Singular integrals and differentiability properties of functions. Princeton, New Jersey: Princeton University Press 1970 
